\title{
Microscopic nonequilibrium theory of double-barrier Josephson junctions
}

\author{
A. Brinkman, A. A. Golubov, and H. Rogalla \\ Faculty of Science and Technology and MESA+Research Institute, University of Twente, 7500 AE, Enschede, The Netherlands \\ F. K. Wilhelm \\ Sektion Physik and CeNS, Ludwig-Maximilians-Universität, Theresienstr. 37, D-80333 München, Germany
}

M. Yu. Kupriyanov

Institute of Nuclear Physics, Moscow State University, 119899 Moscow, Russia

(Received 31 July 2003; published 19 December 2003)

\begin{abstract}
We study nonequilibrium charge transport in a double-barrier Josephson junction, including nonstationary phenomena, using the time-dependent quasiclassical Keldysh Green's function formalism. We supplement the kinetic equations by appropriate time-dependent boundary conditions and solve the time-dependent problem in a number of regimes. From the solutions, current-voltage characteristics are derived. It is understood why the quasiparticle current can show excess current as well as deficit current and how the subgap conductance behaves as function of junction parameters. A time-dependent nonequilibrium contribution to the distribution function is found to cause a nonzero averaged supercurrent even in the presence of an applied voltage. Energy relaxation due to inelastic scattering in the interlayer has a prominent role in determining the transport properties of double-barrier junctions. Actual inelastic scattering parameters are derived from experiments. It is shown as an application of the microscopic model, how the nature of the intrinsic shunt in double-barrier junctions can be explained in terms of energy relaxation and the opening of Andreev channels.
\end{abstract}

DOI: 10.1103/PhysRevB.68.224513 PACS number(s): 74.45.+c, 74.40.+k, 74.25.Fy, 74.50.+r

\section{INTRODUCTION}

The Josephson effect is a hallmark of superconductivity. It is also the basis of a wide range of applications such as metrology and sensing and classical and quantum logic circuits. Moreover, a detailed study of the Josephson effect in mesoscopic devices provides deep insight into the mechanism of the formation and transport of superconducting correlations in weak links and at interfaces. It is known from the microscopic theory of superconductivity that the supercurrent across weak links is carried by Andreev bound states (ABS's). The supercurrent depends both on the ABS energy levels and on their population, i.e. on the quasiparticle distribution function over energy. This provides the possibility of an external control of current. It was realized a long time ago that deviations from equilibrium may strongly modify the transport properties of weak links and Josephson tunnel junctions. A review of early work on various aspects of nonequilibrium superconductivity was given by the papers in Refs. 1 and 2 and the one by Kopnin. ${ }^{3}$ Two main topics in this field are the effects arising from a charge imbalance ${ }^{4,5}$ and effects from a stimulation of superconductivity by external fields. ${ }^{6}$

The recent progress in the fabrication of superconducting structures of submicrometer size has stimulated a renewed interest in nonequilibrium effects in Josephson junctions. The effect of supercurrent control by current injection from additional terminals was first studied theoretically ${ }^{7-9}$ and demonstrated experimentally ${ }^{10,11}$ for a diffusive superconductor-normal metal-superconductor (SNS) junction. In this case, even a sign reversal of the critical current is possible. ${ }^{8,11}$ Additionally, the control of the supercurrent by current injection was studied in structures with ballistic transport. ${ }^{12-17}$

Deviations from equilibrium are also reflected in the quasiparticle current. The dissipative current component in SNS junctions arises from multiple Andreev reflections (MAR) of quasiparticles. A quasiparticle gains $e V$ in energy each time it traverses the interlayer, resulting in a strong nonequilibrium distribution function at subgap energies, as observed by Pierre et al. ${ }^{18}$ In point contacts, the microcscopic description of the current in terms of MAR was derived by Averin and Bardas, ${ }^{19}$ based on a scattering matrix approach, while Cuevas et al. ${ }^{20}$ described MAR in quantum point contacts by means of a tunnel Hamiltonian approach. For the case of an SNS junction with a long interlayer as compared to the coherence length (incoherent regime), the current due to MAR was calculated by Bezuglyi et al. ${ }^{21}$

When tunnel barriers $(I)$ are introduced at the SN interfaces, the quasiparticles in short junctions can undergo transmission resonances, resulting in a dephasing of the electrons and holes. However, it was shown in Ref. 22 that for a broad transmission resonance in a superconductor-insulator-normal metal-insulator-superconductor (SINIS) junction, the resonance energy width being larger than the ABS, coherent transport occurs, and a microscopic model was given in terms of MAR, integrated by a universal distribution of transparency eigenvalues. ${ }^{23}$ It is shown by Naveh et al. ${ }^{24}$ that the same distribution function also describes electrical transport in high critical current density junctions. In order to model the nonstationary and nonequilibrium transport through SINIS structures in the general case, a full Keldysh Green's function approach is required. The derivation of this microscopic model as well as its solutions is the scope of this article.

SINIS junctions are promising basic elements for applica- 
tions in classical computing and metrology, because they are intrinsically shunted. Both rapid single flux quantum logic ${ }^{25}$ and digital voltage standards are electronic applications for which the use of these structures seems promising. ${ }^{26}$ However, important features of the $I V$ characterstics are not sufficiently understood yet, such as the magnitude of the subgap conductance and the nature of the intrinsic shunt of these junctions. The experimental observation that the hysteresis in the $I V$ curves depends nonmonotonically on the critical current density has not yet been explained. ${ }^{26,27}$ Thus, understanding the transport properties on a microscopic level will be valuable for electronic applications. Here, these transport phenomena will be clarified in terms of the opening of Andreev channels and inelastic scattering in the interlayer.

Earlier work concentrated on modeling the $I V$ characteristics of double-barrier junctions in specific limiting cases. When one of the electrodes is replaced by a normal metal, the time dependencies simplify considerably, since formally one can then put the voltage to zero in the superconductor. The $I V$ characteristics of superconductor-insulator-normal metal-insulator-normal metal (SININ) junctions were studied by means of the quasiclassical Green's functions technique by Zaitsev, ${ }^{28}$ Volkov et al.,${ }^{29}$ and Zaitsev et al.${ }^{30}$ Lempitskii ${ }^{31}$ studied nonequilibrium effects on the nonstationary properties of long SNS junctions in the absence of interface barriers and $\operatorname{Kadin}^{32}$ used a time-dependent Ginzburg-Landau approach, valid only in a narrow temperature range. Another limiting case is the double-barrier structure with a long interlayer as compared to the coherence length. The derivation of time-dependent transport properties in this case simplifies since a decoupling of the electrodes is possible, as, e.g., studied by Volkov and Klapwijk ${ }^{33}$ and Bezuglyi et al. ${ }^{21}$

In this paper, a microscopic quasiclassical theory will be given for a double-barrier Josephson junction with two superconducting electrodes and a short interlayer. The interlayer will be assumed to be a diffusive normal metal, but it will be indicated how the model can be extended in a straightforward way to incorporate a superconducting gap in the interlayer. The Keldysh formalism is introduced in Sec. II. The spectral supercurrent density is obtained, and appropriate time-dependent boundary conditions are derived to supplement the kinetic equations for the energy distribution functions in the interlayer. The technical scheme for solving the time-dependent Keldysh-Usadel equation may have applications beyond the present paper. Solutions are presented in Sec. III for the adiabatic limit of $e V \ll \Delta_{S}$. As an intriguing nonequilibrium effect in a double-barrier Josephson junction, we show that even at finite voltage bias, there can be a nonzero averaged supercurrent. Energy relaxation due to inelastic scattering is a phenomenon that strongly modifies the energy distribution function. It will be shown in Sec. IV that inelastic scattering is important in a double-barrier Josephson junction and how this effect can be incorporated into the microscopic model. As an application of the microscopic model, the nature of the intrinsic shunt of double-barrier junctions will be discussed in Sec. V. The observed nonmonotonic hysteresis vs critical current density dependence, as well as the actual values, are explained.

\section{KELDYSH FORMULATION}

The Matsubara Green's function technique can be applied to a many-body system in equilibrium, from which the energy-dependent properties of the system can be derived. In addition to obtaining spectral quantities, we need to know how the states are populated under nonequilibrium conditions. For this purpose Keldysh ${ }^{34}$ proposed a set of propagators along a contour in the complex-time plane that allows one to describe the real-time evolution of a system outside equilibrium and at a finite temperature. The review of Rammer and Smith ${ }^{35}$ describes the use of the Keldysh technique in the transport theory of metals. The Keldysh method is introduced specifically for nonequilibrium superconductivity in Refs. 36-39.

The quasiclassical approximation is used, in the sense that rapid oscillations of the wave functions on the scale of the Fermi wavelength are averaged out. Furthermore, in this paper it is assumed that the transport through the interlayer is diffusive, the thickness being much larger than the elastic scattering length, so that the Usadel equation can be used.

\section{A. Time-dependent Usadel equation}

A compact notation of the equations for the quasiclassical Green's functions becomes possible by introducing the Green's function in Keldysh $\times$ Nambu space:

$$
\breve{G}=\left(\begin{array}{cc}
\hat{G}^{R} & \hat{G}^{K} \\
0 & \hat{G}^{A}
\end{array}\right) .
$$

The quasiclassical Green's function $\breve{G}$ is a function of two times, $t$ and $t^{\prime}$, and the time-dependent Usadel equation in the absence of a vector potential reads ${ }^{38}$

$$
\begin{aligned}
&-\mathcal{D} \hbar \nabla(\breve{G} \circ \nabla \breve{G})+\breve{\tau_{3}} \hbar \frac{\partial \breve{G}}{\partial t}+\frac{\partial \breve{G}}{\partial t^{\prime}} \hbar \breve{\tau}_{3}-i \breve{\Delta}(t) \breve{G}+\breve{G} i \breve{\Delta}\left(t^{\prime}\right) \\
&=-i\left(\breve{\Sigma}_{\text {inel }}^{\circ} \breve{G}-\breve{G} \circ \breve{\Sigma}_{\text {inel }}\right)
\end{aligned}
$$

where

$$
\begin{gathered}
\breve{\tau}_{3}=\left(\begin{array}{cc}
\hat{\tau}_{3} & 0 \\
0 & \hat{\tau}_{3}
\end{array}\right), \breve{\Delta}=\left(\begin{array}{cc}
\hat{\Delta} & 0 \\
0 & \hat{\Delta}
\end{array}\right), \\
\hat{\Delta}=\left(\begin{array}{cc}
0 & \Delta \\
\Delta * & 0
\end{array}\right), \breve{\Sigma}_{\text {inel }}=\left(\begin{array}{cc}
\hat{\Sigma}_{\text {inel }} & 0 \\
0 & \hat{\Sigma}_{\text {inel }}
\end{array}\right) .
\end{gathered}
$$

$\mathcal{D}$ is the diffusion constant, $\breve{\Sigma}_{\text {inel }}$ the self-energy with retarded, advanced, and Keldysh components, * denotes the complex conjugate and $\circ$ denotes a convolution over the internal time coordinates, e.g., $\breve{\Sigma}_{\text {inel }}\left(t, t^{\prime}\right) \circ \breve{G}$ $=\int d t_{1} \breve{\Sigma}_{\text {inel }}\left(t, t_{1}\right) \breve{G}\left(t_{1}, t^{\prime}\right)$. The function $\breve{G}$ is normalized as $\breve{G} \breve{G}^{\circ}=1$. The expression for the current in the Keldysh formalism is

$$
I=\frac{1}{2 e R_{N}} \int d E \operatorname{Tr}\left[\hat{\tau}_{3}\left(\hat{G}^{R} \nabla \hat{G}^{K}+\hat{G}^{K} \nabla \hat{G}^{A}\right)\right] .
$$


The Green's functions can be transformed to energyfrequency space $(E, \omega)$ by Fourier transforming the functions $\breve{G}\left(t-t^{\prime},\left(t+t^{\prime}\right) / 2\right)$ :

$$
\begin{aligned}
\breve{G}(E, \omega)= & \int \breve{G}\left(t-t^{\prime}, \frac{t+t^{\prime}}{2}\right) \\
& \times e^{-i E\left(t-t^{\prime}\right) / \hbar} e^{i \omega\left(t+t^{\prime}\right) / 2 \hbar} d\left(t-t^{\prime}\right) d\left(t+t^{\prime}\right) / 2 .
\end{aligned}
$$

This transformation is analogous to the Wigner representation of the full double-coordinate Green's function. Spectral quantities that only depend on energy and not on frequency after Fourier transforming, such as the equilibrium Green's functions in the electrodes, only depend on the time difference before Fourier transforming. Each term in Eq. (2) can be transformed to $(E, \omega)$ space. Hence, the Usadel equation can be rewritten in $(E, \omega)$ space as

$$
\begin{aligned}
-\mathcal{D} & \hbar\left((\breve{G} \circ \nabla \breve{G})+i E\left[\breve{\tau}_{3}, \circ \breve{G}\right]+i \frac{\omega}{2}\left\{\breve{\tau}_{3}, \circ \breve{G}\right\}\right. \\
= & -i\left(\breve{\Sigma}_{\text {inel }}^{\circ} \breve{G}-\breve{G} \circ \breve{\Sigma}_{\text {inel }}\right),
\end{aligned}
$$

where $\hat{\Delta}(t)=0$ is taken for simplicity. $\left[\breve{\tau}_{3}, \breve{G}\right]$ is the commutator of $\breve{\tau}_{3}$, and $\breve{G}$, and $\left\{\breve{\tau}_{3}, \breve{G}\right\}$ is the anticommutator. A decomposition of the Green's functions in Fourier harmonics can formally be introduced as

$$
\breve{G}(E, \omega)=\sum_{n=-\infty}^{\infty} \breve{G}_{n}(E) \delta\left(\omega-\omega_{0}\right),
$$

where $\breve{G}_{n}(E)=\breve{G}\left(E, n \omega_{0}\right)$, as was done, for example in Ref. 20. The generation of higher order Fourier harmonics is a manifestation of the nonlinearity of the device, prevalent, e.g., in Eq. (6).

\section{B. Retarded and advanced propagators}

Equation (6) consists of an Usadel equation for the retarded Green's function, the advanced Green's function, and an equation containing the Keldysh Green's function. The Usadel equation for the retarded Green's function $\hat{G}^{R}$ in the interlayer (taking the limit of $\hat{\Delta}=0$ and zero inelastic scattering, $\hat{\Sigma}_{\text {inel }}=0$ ) in Fourier components reads

$$
\begin{aligned}
& -\mathcal{D} \hbar \nabla\left(\hat{G}^{R} \circ \nabla \hat{G}^{R}\right)_{n}+i n \omega_{0} / 2\left\{\hat{\tau}_{3}, \hat{G}_{n}^{R}(E)\right\}+i E\left[\hat{\tau}_{3}, \hat{G}_{n}^{R}(E)\right] \\
& \quad=0,
\end{aligned}
$$

where the index $n$ denotes the $n$th harmonic. The self-energy terms in Eq. (6) can effectively be represented by a characteristic inelastic scattering time $\tau_{\text {in }}$, as derived by Larkin and Ovchinnikov. ${ }^{40}$ Taking the inelastic scattering to be time independent is of course a rough approximation, but suffices for the purposes of this paper. The self-energy terms have been neglected in Eq. (8), which is justified as long as $\hbar / \tau_{i n} \ll k_{B} T$. Note that Eq. (8) can be reduced to the timeindependent case for $n=0$. Time dependence occurs if the

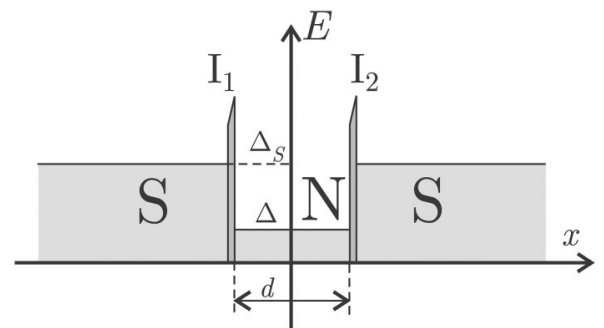

FIG. 1. Schematic representation of the double-barrier SINIS structure. Two superconducting electrodes (S) are separated by two delta-shaped potential barriers $\left(\mathrm{I}_{1}\right.$ and $\left.\mathrm{I}_{2}\right)$ and a normal metal interlayer $(\mathrm{N})$. The position dependence of the pair potential has been indicated by shading.

boundary conditions, which will be detailed below, provide nonzero limiting values for $\hat{G}_{n}$.

The most general decomposition of the retarded and advanced Green's functions is a linear combination of the three Pauli matrices. ${ }^{35}$ Whenever the phase is constant, it can be chosen such that it suffices to define

$$
\begin{gathered}
\hat{G}^{R(A)}=G^{R(A)} \hat{\tau}_{3}+F^{R(A)} \hat{\tau}_{1}, \\
\hat{G}^{A}=-\hat{\tau}_{3} \hat{G}^{R \dagger} \hat{\tau}_{3}=-G^{R *} \hat{\tau}_{3}+F^{R *} \hat{\tau}_{1} .
\end{gathered}
$$

Assuming that the thickness of the interlayer $d$ is much smaller than the coherence length in the interlayer, $\xi$ $=\sqrt{\mathcal{D} / E}$ where $E$ is a characteristic energy at which the system is probed, we can take the retarded Green's function to be much larger than its gradient. The double-barrier structure under consideration is depicted in Fig. 1. Integrating both sides of Eq. (8) over the interlayer thickness and barriers gives

$$
\begin{aligned}
& \left.\hbar \mathcal{D}\left(\hat{G}^{R} \circ \nabla \hat{G}^{R}\right)_{n}\right|_{x=0^{+}}-\left.\hbar \mathcal{D}\left(\hat{G}^{R} \circ \nabla \hat{G}^{R}\right)_{n}\right|_{x=d^{-}} \\
& \quad+i n \frac{\omega_{0}}{2} d\left\{\hat{\tau}_{3}, \hat{G}_{n}^{R}(E)\right\}+i E d\left[\hat{\tau}_{3}, \hat{G}_{n}^{R}(E)\right]=0 .
\end{aligned}
$$

Zaitsev $^{41}$ derived effective boundary conditions for the quasiclassical Green's function formalism. These were further developed for diffusive scattering in the interlayer by Kupriyanov and Lukichev. ${ }^{42}$ Using the KupriyanovLukichev boundary conditions for the retarded Green's functions,

$$
\left.\xi \gamma_{B}\left(\hat{G}^{R} \circ \nabla \hat{G}^{R}\right)_{n}\right|_{x=0, d}= \pm\left[\hat{G}^{R}, \circ \hat{G}_{S_{L, R}}^{R}\right]_{n},
$$

where $\gamma_{B}=R_{B} / \rho \xi, \rho$ is the resistivity of the interlayer, and $R_{B}$ is the interface resistance, we obtain

$$
\begin{aligned}
& \text { in } \frac{\omega_{0}}{2}\left\{\hat{\tau}_{3}, \hat{G}_{n}^{R}(E)\right\} \gamma_{B} d / \xi+i E\left[\hat{\tau}_{3}, \hat{G}_{n}^{R}(E)\right] \gamma_{B} d / \xi \\
& \quad+2 \pi k_{B} T_{c S}\left[\hat{G}^{R}, \circ\left(\hat{G}_{S_{R}}^{R}+\hat{G}_{S_{L}}^{R}\right)\right]_{n}=0,
\end{aligned}
$$

where $\hat{G}_{S_{L, R}}^{R}$ are retarded functions in the left and right electrodes, respectively. The normalization condition for $\hat{G}^{R}$ in 
energy-space and decomposed into Fourier harmonics can be found from the expressions of Appendix B:

$$
\delta_{n 0}=\sum_{m=-\infty}^{\infty} \hat{G}_{m}^{R}\left(E+\frac{n-m}{2} \omega_{0}\right) \hat{G}_{n-m}^{R}\left(E-\frac{m}{2} \omega_{0}\right) .
$$

Equations (12) and (13) form a complete set of equations from which the Fourier components of $\hat{G}^{R}$ can in principle be determined. This recursive schemes reflects the fact, that superconducting correlations can be induced over several MAR cycles. Solving the set of equations is complicated by the recurrent nature of the equations. The Fourier harmonics are coupled to each other and have arguments that are shifted in energy.

From the full set of equations, we can find back the quasistationary Matsubara case by keeping only the $n=0$ harmonic of $G^{R}$ and the $n= \pm 1$ harmonics of $F^{R}$ and neglecting energy shifts in the arguments. This provides a solution for $G^{R}$ and $F^{R}$ that coincides with the analytical continuation $(\omega \rightarrow-i E)$ of the Matsubara solution at $\varphi=\pi / 2$. The general Matsubara solutions for double-barrier junctions with $\gamma_{B 1,2} \gg 1$ and $d / \xi \ll 1$ were obtained in Ref. 26 . In the limit of $\Delta=0$ the analytical continuation $\omega \rightarrow-i E$ of this solution provides the Green's a functions at $T=0$ as a function of energy,

$$
\begin{gathered}
\Phi=\frac{E F_{S}}{E \gamma_{\mathrm{eff}} / \pi k_{B} T_{c}+i G_{S}}\left(\cos \frac{\varphi}{2}+i \gamma_{-} \sin \frac{\varphi}{2}\right), \\
G=\frac{E}{\left(E^{2}-|\Phi|^{2}\right)}, \quad F=\frac{\Phi}{\left(|\Phi|^{2}-E^{2}\right)},
\end{gathered}
$$

where $F_{S}=\Delta_{S} /\left(\Delta_{S}^{2}-E^{2}\right)$ and $G_{S}=E /\left(E^{2}-\Delta_{S}^{2}\right)$. The asymmetry and effective suppression parameter are, respectively,

$$
\gamma_{-}=\frac{\gamma_{B 1}-\gamma_{B 2}}{\gamma_{B 1}+\gamma_{B 2}}, \quad \gamma_{\mathrm{eff}}=\frac{d}{\xi} \frac{\gamma_{B 1} \gamma_{B 2}}{\gamma_{B 1}+\gamma_{B 2}}
$$

As an illustration to these retarded Green's functions, the density of states in the interlayer, $N=\operatorname{Re} G$, is shown in Fig. 2. It can be seen that for $\gamma_{\mathrm{eff}} \gg 1$ the density of states is determined by a minigap with a value of $\cos (\varphi / 2) \pi k_{B} T_{c} / \gamma_{\text {eff }}$. In the coherent regime 22 of $\gamma_{\text {eff }} \ll 1$ the gap in the density of states is given by $\Delta \cos (\varphi / 2)$. The density of states for intermediate values of the suppression parameter is characterized by a two-peak structure. These findings coincide with the calculations of Bezuglyi et al. $^{45}$ in the limiting case of a short interlayer.

\section{Spectral supercurrent}

Supercurrent is carried by states in the weak link and their occupation is determined by a distribution function. The supercurrent-carrying density of states, or spectral supercurrent $\operatorname{Im} I_{S}(E)$, can be determined from $\hat{G}^{R}$ and $\hat{G}^{A}$ by

$$
\operatorname{Im} I_{S}=\frac{1}{8} \operatorname{Tr}\left[\hat{\tau}_{3}\left(\hat{G}^{R} \circ \nabla \hat{G}^{R}-\hat{G}^{A} \circ \nabla \hat{G}^{A}\right)\right] .
$$

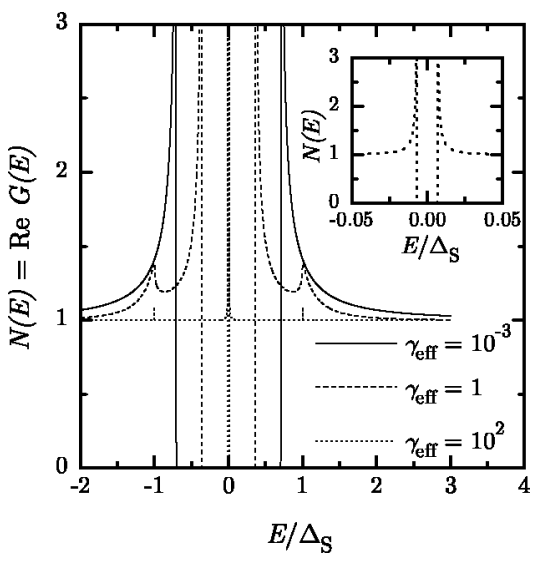

FIG. 2. Normalized density of states in the interlayer at $T=0$ for several values of the suppression parameter $\gamma_{\text {eff }}$. The inset shows the minigap that is present for $\gamma_{\mathrm{eff}}=10^{2}$ on a smaller scale.

The supercurrent in the regime of $\gamma_{\mathrm{eff}} \ll 1$, is found to have a spectral density

$$
\operatorname{Im} I_{S}(E) e R_{N}=\frac{\Delta_{S}^{2} \sin \varphi}{\sqrt{\Delta_{S}^{2}-E^{2}} \sqrt{E^{2}-\Delta_{S}^{2} \cos ^{2}(\varphi / 2)}}
$$

for $\Delta_{S} \cos (\varphi / 2)<E<\Delta_{S}$, while $\operatorname{Im}_{S}(E)=0$ for $E$ $<\Delta_{S} \cos (\varphi / 2)$ and $E>\Delta_{S}$. This universal expression is independent of the interlayer thickness, barrier height, and contact dimensionality as long as the number of conduction channels is large. ${ }^{43}$ The same expression was found in the case of ballistic interlayer transport. ${ }^{22}$ The spectral supercurrent is nonzero only in the range $\Delta_{S} \cos (\varphi / 2)<E<\Delta_{S}$, i.e. there is a minigap $\Delta_{S} \cos (\varphi / 2)$ in the spectrum of the Andreev bound states, see the inset of Fig. 3. On the other hand, all states in the energy range $\Delta_{S} \cos (\varphi / 2)$ contribute to the supercurrent. In long junctions, a similar behavior for the density of states was found in Ref. 44 and for the current in Ref. 8. The contact is in the intermediate regime between a short ballistic SNS weak link, with a bound state energy $\Delta_{S} \cos (\varphi / 2)$ and a tunnel junction with a bound state energy

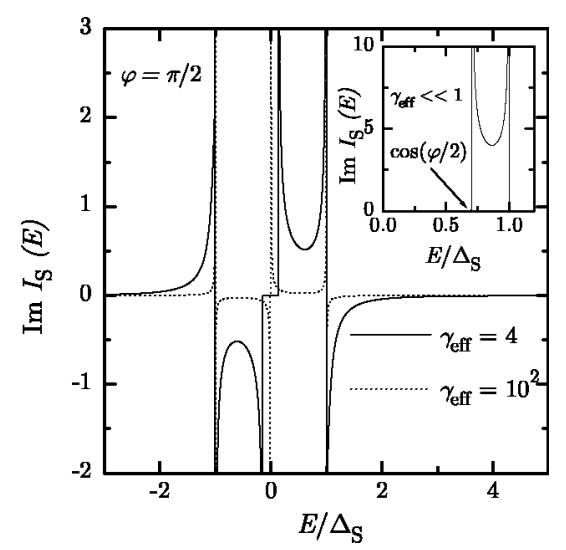

FIG. 3. Normalized spectral supercurrent density as a function of energy for various values of the suppression parameter $\gamma_{\text {eff }}$. The phase difference between the superconducting electrodes was fixed at $\varphi=\pi / 2$. The inset shows the spectral supercurrent in the coherent regime of $\gamma_{\mathrm{eff}} \ll 1$. 
$\Delta_{S}$. Physically this is caused by the properties of the distribution of transparencies, which is a combination of open and closed channels (see Ref. 22).

In the incoherent regime of $\gamma_{\mathrm{eff}} \gg 1$, this universality breaks down. The minigap in the spectrum of Andreev bound states is now given by $\cos (\varphi / 2) \pi k_{B} T_{c} / \gamma_{\text {eff }}$. Figure 3 shows the spectral supercurrent density for several values of the suppression parameter. The sign change at $E=\Delta_{S}$ has also been observed by Bezuglyi et al. ${ }^{45}$ and Heikkilä et al. ${ }^{46}$ Going beyond the approximations $d \ll \xi$ and $\gamma_{B} \ll 1$, it was shown by Schäpers et al. ${ }^{47}$ for ballistic junctions that lowenergy states are gradually filled in for larger interlayer thickness and by a larger barrier transparency.

\section{Kinetic equations}

The energy distribution functions, that determine the occupation of spectral functions, can be determined from the kinetic equations. From the matrix normalization condition $\breve{G} \circ \breve{G}=\breve{1}$, the upper right component implies that $\hat{G}^{R} \circ \hat{G}^{K}$ $+\hat{G}^{K} \circ \hat{G}^{A}=0$. Hence, $\hat{G}^{K}$ can be parametrized as

$$
\hat{G}^{K}=\hat{G}^{R} \circ \hat{f}-\hat{f} \circ \hat{G}^{A} .
$$

Furthermore, it was shown by Schmid and Schön ${ }^{48}$ and Larkin and Ovchinnikov ${ }^{40}$ that $\hat{f}$ can be chosen to be diagonal. We will adopt the notation

$$
\hat{f}=f_{L} \hat{1}+f_{T} \hat{\tau}_{3},
$$

where $f_{L}$ and $f_{T}$ are those parts of the distribution function that are respectively even and odd in energy. Therefore they are named longitudinal and transverse energy distribution function respectively. The functions can be identified with energy and particle flow. ${ }^{49}$ Physically, a deviation of $f_{L}$ from equilibrium is associated with a different effective temperature and a deviation of $f_{T}$ from equilibrium with a chemical potential shift. In equilibrium, $f_{T 0}=0$ and $f_{L 0}$ $=\tanh \left(E / 2 k_{B} T\right)$.

Putting Eqs. (18) and (19) into the Keldysh component of the Usadel equation (2) and by making use of the Usadel equations for the retarded and advanced Green's function, finally the kinetic equations for the Fourier components of $f_{L}$ and $f_{T}$ can be written as

$$
\begin{aligned}
\left(D_{L} \circ \nabla^{2} f_{L}\right)_{n}+\left(\operatorname{Im} I_{S} \circ \nabla f_{T}\right)_{n}= & \frac{1}{\hbar \mathcal{D}}\left(n i \omega_{0}+\hbar \tau_{i n}^{-1}\right) \\
& \times\left[G^{R} \circ\left(f_{L}-f_{0} \delta_{n 0}\right)\right. \\
& \left.-\left(f_{L}-f_{0} \delta_{n 0}\right) G^{A}\right]_{n}, \\
\left(D_{T^{\circ}} \nabla^{2} f_{T}\right)_{n}+\left(\operatorname{Im} I_{S} \circ \nabla f_{L}\right)_{n}= & \frac{1}{\hbar \mathcal{D}}\left(n i \omega_{0}+\hbar \tau_{i n}^{-1}\right) \\
& \times\left(G^{R} \circ f_{T}-f_{T^{\circ}} G^{A}\right)_{n},
\end{aligned}
$$

with the generalized transverse and longitudinal diffusion coefficients being $4 D_{T}=\operatorname{Tr}\left(1-\hat{\tau}_{3} \hat{G}^{R} \circ \hat{\tau}_{3} \hat{G}^{A}\right)$ and $4 D_{L}=\operatorname{Tr}(1$ $\left.-\hat{G}^{R} \circ \hat{G}^{A}\right)$. With the parametrization of Eq. (9) this can be further rewritten as $D_{T}=(\operatorname{Re} G)^{2}+(\operatorname{Re} F)^{2}$ and $D_{L}$ $=(\operatorname{Re} G)^{2}-(\operatorname{Im} F)^{2}$. In obtaining Eqs. (20), use has been made of the rewriting of the $\breve{\Sigma} \circ \breve{G}-\breve{G} \circ \breve{\Sigma}$ term by Larkin and Ovchinnikov ${ }^{40}$ into a collision integral with characteristic inelastic scattering time $\tau_{i n}$. $\Delta$ has been assumed to be negligible for simplicity, but a superconducting gap in the interlayer can be incorporated into the model in a straightforward way by keeping the terms in the Usadel equation (2) that depend on $\Delta$. In the limit of slow time variations, a Fourier transform over the time difference provides the known mixed representation of the kinetic equations ${ }^{38}$

$$
\begin{gathered}
\mathcal{D} D_{L} \nabla^{2} f_{L}+\mathcal{D} \operatorname{Im} I_{S} \nabla f_{T}-\operatorname{Re} G\left(\tau_{\text {in }}^{-1}+\frac{d}{d t}\right)\left(f_{L}-f_{0}\right)=0, \\
\mathcal{D} D_{T} \nabla^{2} f_{T}+\mathcal{D} \operatorname{Im} I_{S} \nabla f_{L}-\operatorname{Re} G\left(\tau_{i n}^{-1}+\frac{d}{d t}\right) f_{T}=0,
\end{gathered}
$$

which follows directly from Eq. (20) for the lowest Fourier harmonic. The expressions for the supercurrent and dissipative current components can be derived ${ }^{39}$ from Eq. (4)

$$
\begin{aligned}
& I_{S}=\frac{1}{2 e R_{N}} \int d E f_{L}(E) \operatorname{Im} I_{S}(E), \\
& I_{N}=\frac{1}{2 e R_{N}} \int d E D_{T}(E) \nabla f_{T}(E) .
\end{aligned}
$$

What remains to be derived is a proper set of time-dependent boundary conditions for the kinetic equations.

\section{E. Time-dependent boundary conditions}

The Kupriyanov-Lukichev boundary conditions ${ }^{42}$ for the quasiclassical Green's functions can in general be written as

$$
\gamma_{B} \xi \breve{G} \circ \frac{d}{d x} \breve{G}=\breve{G} \circ \breve{G}_{1}-\breve{G}_{1} \circ \breve{G},
$$

where $\breve{G}_{1}$ and $\breve{G}$ denote the Green's functions at the two sides of the first interface. From the definition of the Green's functions in Keldysh space, Eq. (1), a boundary condition can be written for each matrix element. In Appendix A, this set of boundary conditions is rewritten into

$$
\begin{aligned}
\gamma_{B} \xi\left[\left(1-\hat{G}^{R} \hat{G}^{A}\right) \frac{d}{d x} f_{L}+\left(\hat{\tau}_{3}-\hat{G}^{R} \hat{\tau}_{3} \hat{G}^{A}\right) \frac{d}{d x} f_{T}\right] \\
=\left[\hat{G}^{R}\left(\hat{G}_{1}^{R}-\hat{G}_{1}^{A}\right)-\left(\hat{G}_{1}^{R}-\hat{G}_{1}^{A}\right) \hat{G}^{A}\right]\left(f_{L 1}-f_{L}\right) \\
\quad+\left[\hat{G}^{R}\left(\hat{G}_{1}^{R} \hat{\tau}_{3}-\hat{\tau}_{3} \hat{G}_{1}^{A}\right)-\left(\hat{G}_{1}^{R} \hat{\tau}_{3}-\hat{\tau}_{3} \hat{G}_{1}^{A}\right) \hat{G}^{A}\right] \\
\quad \times\left(f_{T 1}-f_{T}\right),
\end{aligned}
$$

where all the products have to be regarded as time convolutions and $f_{L 1 / T 1}$ are the distribution functions in the respective reservoir.

The Green's functions become time dependent by applying a voltage over the interface. In the absence of voltage, the Green's functions in the electrodes only depend on the time difference since equilibrium is assumed. The potential 
can be introduced in each electrode by a gauge transformation $^{41}$ of the Green's function in the electrodes

$$
\hat{G}_{1}^{R(A)}\left(t, t^{\prime}\right)=\hat{S}(t) \hat{G}_{1}^{R(A)}\left(t-t^{\prime}\right) \hat{S}^{\dagger}\left(t^{\prime}\right),
$$

where $\hat{S}(t)$ and $\hat{S}^{\dagger}\left(t^{\prime}\right)$ are given by

$$
\begin{aligned}
S(t) & =\left(\begin{array}{cc}
e^{i e V t / \hbar} & 0 \\
0 & e^{-i e V t / \hbar}
\end{array}\right), \\
S^{\dagger}\left(t^{\prime}\right) & =\left(\begin{array}{cc}
e^{-i e V t^{\prime} / \hbar} & 0 \\
0 & e^{i e V t^{\prime} / \hbar}
\end{array}\right) .
\end{aligned}
$$

Volkov and Klapwijk ${ }^{33}$ performed a gauge transformation of the interlayer Green's functions, which works only in the limit $d \gg \xi$ because of the small coupling between the electrodes in this case, which allows one to neglect the interference terms in the interlayer leading to a local time dependence.

By performing the gauge transformation for $\hat{G}_{1}^{R}$ and $\hat{G}_{1}^{A}$ and by taking the trace from Eq. (25), one obtains the first boundary condition in time representation. The second equation is obtained by taking the trace after multiplying left- and right-hand sides of Eq. (25) by $\hat{\tau}_{3}$. This results in

$$
\begin{aligned}
D_{T} \gamma_{B} \xi \frac{d}{d x} f_{T}= & \left\{\operatorname{Re} G_{1} \operatorname{Re} G i \sin \left[\frac{e V}{\hbar}\left(t-t^{\prime}\right)\right]\right. \\
& \left.+\operatorname{Im} F_{1} \operatorname{Re} F \sin \left[\frac{e V}{\hbar}\left(t+t^{\prime}\right)\right]\right\}\left(f_{L 0}-f_{L}\right) \\
& -f_{T}\left\{\operatorname{Re} G_{1} \operatorname{Re} G \cos \left[\frac{e V}{\hbar}\left(t-t^{\prime}\right)\right]\right. \\
& \left.+\operatorname{Re} F_{1} \operatorname{Re} F \cos \left[\frac{e V}{\hbar}\left(t+t^{\prime}\right)\right]\right\} \\
D_{L} \gamma_{B} \xi \frac{d}{d x} f_{L}= & \left\{\operatorname{Re} G_{1} \operatorname{Re} G \cos \left[\frac{e V}{\hbar}\left(t-t^{\prime}\right)\right]\right. \\
& \left.-\operatorname{Im} F_{1} \operatorname{Im} F \cos \left[\frac{e V}{\hbar}\left(t+t^{\prime}\right)\right]\right\}\left(f_{L 0}-f_{L}\right) \\
& -f_{T}\left\{\operatorname{Re} G_{1} \operatorname{Re} G i \sin \left[\frac{e V}{\hbar}\left(t-t^{\prime}\right)\right]\right. \\
& \left.+\operatorname{Re} F_{1} \operatorname{Im} F \sin \left[\frac{e V}{\hbar}\left(t+t^{\prime}\right)\right]\right\}
\end{aligned}
$$

where all products are time convolutions and use has been made of the fact that $f_{T 1}=0$, since the electrodes are assumed to be in internal equilibrium. The energy distribution functions are not only coupled through the kinetic equations (20), but through the boundary conditions as well.

At the second interface a similar set of boundary conditions can be derived, which can be obtained from Eqs. (28) and (29) by replacing $G_{1}$ and $F_{1}$ by $G_{2}$ and $F_{2}$, respectively, and by multiplying the right-hand side of Eqs. (28) and (29) by -1 .
Note that $D_{L}=(\operatorname{Re} G)^{2}-(\operatorname{Im} F)^{2}=0$ for energies smaller than the minigap in the interlayer. Hence, for energies at which $D_{L}=0$, boundary condition (29) is replaced by $f_{L}$ $=f_{L 0}$. This physically means that the system does not conduct heat inside the gap and that the distribution in the gap is controlled by coupling to some external heat bath, e.g., through the substrate, and not through the superconducting leads.

Each term in the boundary condition contains time convolutions. With the aid of the expansion of the Green's functions in Fourier harmonics and the expressions of Appendix $\mathrm{B}$ for the time convolutions of double and triple products, the convolutions can be worked out for each term. The left-hand side of Eq. (28) is, for example,

$$
\begin{gathered}
D_{T^{\circ}} \gamma_{B} \xi \frac{d}{d x} f_{T}=\sum_{n, n^{\prime}} \int_{-\infty}^{\infty} D_{T, n}\left(E+n^{\prime} \omega_{0} / 2\right) \gamma_{B} \xi \\
\frac{d}{d x} f_{T, n^{\prime}}\left(E-n \omega_{0} / 2\right) e^{i E\left(t-t^{\prime}\right) / \hbar} e^{i\left[\left(n+n^{\prime}\right) / 2 \hbar\right] \omega_{0}\left(t+t^{\prime}\right)} d E .
\end{gathered}
$$

The sine and cosine dependencies in the boundary conditions cause additional voltage shifts as well as coupling to higher harmonics, which can be seen, for example, in the term

$$
\begin{aligned}
f_{L} \circ[ & \left.\operatorname{Re} G_{S} \circ \operatorname{Re} G \circ i \sin \left(t-t^{\prime}\right)\right] \\
= & \sum_{n, n^{\prime}} \int d E f_{L, n}\left(E+n^{\prime} \omega_{0} / 2\right) \operatorname{Re} G_{n^{\prime}}\left(E-n \omega_{0} / 2\right) \\
& \times e^{i E^{\prime}\left(t-t^{\prime}\right) / \hbar} e^{i\left[\left(n+n^{\prime}\right) / 2 \hbar\right] \omega_{0}\left(t+t^{\prime}\right)} \\
& \times \frac{1}{2}\left[\operatorname{Re} G_{S}\left(E+\frac{n^{\prime}-n-1}{2} \omega_{0}\right)\right. \\
& \left.+\operatorname{Re}_{S}\left(E+\frac{n^{\prime}-n+1}{2} \omega_{0}\right)\right]
\end{aligned}
$$

In principle, the set of kinetic equations (20) together with the boundary conditions Eqs. (28) and (29), and expressions for the time convolutions, such as Eq. (30) and Eq. (31), now provide a complete set of equations to solve the energy distribution functions as function of voltage. However, the coupling to higher harmonics and energy shifts within the functions themselves make solving the equations cumbersome. In principle, a solution should crossover to the solutions as found by the MAR approach ${ }^{22}$ in the limit of $\gamma_{\text {eff }} \ll 1$. In Sec. III an adiabatic approximation will be developed in order to solve the kinetic equations for $e V \ll \Delta_{S}$ and a larger suppression parameter.

\section{ADIABATIC DYNAMICS}

\section{A. Adiabatic approximation}

In order to simplify the time dependencies, an adiabatic approximation can be made. When the voltage is small, the phase oscillates slowly and can even be considered quasistationary. In this case, we only need to keep the time dependence in expressions that contain the phase, but can neglect 
all other time dependencies. Consequently, the time convolutions become simple products and the energy shifts can be neglected. Therefore, this approximation is called adiabatic.

A formal derivation of the parameter regime in which the adiabatic approximation can be used, is based on the time dependence in Eqs. (28) and (29). The quasiparticle current is determined by the left-hand side of Eq. (28), namely, $D_{T} \gamma_{B} \xi d f_{T} / d x$. It will be shown in this section that the righthand side of this boundary condition in the adiabatic limit is equal to the $f_{T}$ terms in Eq. (28). Hence, deviations from the adiabatic approximation in the quasiparticle current are only to be expected when the terms proportional to $f_{L}$ in Eq. (28) are not negligible. The first of these terms is $f_{L} \operatorname{Re} G_{1} \operatorname{Re} G i \sin \left[e V\left(t-t^{\prime}\right) / \hbar\right]$, which can be neglected for $e V \ll \Delta_{S}$. The second term is $f_{L} \operatorname{Im} F_{1} \operatorname{Re} F \sin \left[e V\left(t+t^{\prime}\right) / \hbar\right]$, which is nonzero only due to the looplike construction with Eq. (29), in which the terms $\operatorname{Im} F_{1}$ and $\operatorname{Re} F$ are shifted $e V / 2$ in energy every cycle, making their overlap nonzero after approximately $\Delta_{S} / e V$ cycles. For large suppression parameters, $\operatorname{Re} F \sim \gamma_{\text {eff }}^{-1}$. Hence, smallness of this term can now be formulated as $\left(1 / \gamma_{\mathrm{eff}}\right)^{\Delta_{S}} / e V \ll 1$. Combining the conditions, the conclusion is reached that the adiabatic approximation is valid when $e V \ll \Delta_{S}$ and $\gamma_{\mathrm{eff}} \gg 1$.

In this case, the phases $\chi_{1,2}$ can be introduced by the parametrization

$$
\hat{G}_{1,2}^{R}=\left(\begin{array}{cc}
G_{1,2}^{R} & F_{1,2}^{R} \mathrm{e}^{i \chi_{1,2}} \\
F_{1,2}^{R} \mathrm{e}^{-i \chi_{1,2}} & -G_{1,2}^{R}
\end{array}\right) .
$$

No additional gauge transformations have to be performed in the boundary conditions. Hence, we can use the parametrization of Eq. (32) directly in the boundary condition (25). The first boundary condition is then found by taking the trace of Eq. (25). The second is found by taking the trace after multiplying with $\hat{\tau}_{3}$. With Eq. (32) and after some rewriting, this gives

$$
\begin{aligned}
& \gamma_{B 1,2} \xi D_{T} \frac{d}{d x} f_{T}(0, d)= \pm M_{T 1,2}\left[f_{T}(0, d) \mp f_{T 0}\right], \\
& \gamma_{B 1,2} \xi D_{L} \frac{d}{d x} f_{L}(0, d)= \pm M_{L 1,2}\left[f_{L}(0, d)-f_{L 0}\right],
\end{aligned}
$$

where

$$
f_{L 0, T 0}=\frac{1}{2} \tanh \frac{E+e V / 2}{2 k_{B} T} \pm \frac{1}{2} \tanh \frac{E-e V / 2}{2 k_{B} T},
$$

are the distribution functions in the leads and $M_{T 1,2}$ $=\operatorname{Re} G_{S_{L, R}} \operatorname{Re} G+\operatorname{Re} F_{S} \operatorname{Re} F \cos \left(\chi_{1}-\chi_{2}\right), M_{L 1,2}=\operatorname{Re} G_{S_{L, R}} \operatorname{Re} G$ $-\operatorname{Im} F_{S} \operatorname{Im} F \cos \left(\chi_{1}-\chi_{2}\right)$, where $\chi_{1}-\chi_{2}=\varphi / 2=e V t$ in the case of symmetric barriers. Here $G$ and $F$ are given by Eq. (14) and

$$
G_{S_{L, R}}=\frac{E \pm e V / 2}{\sqrt{(E \pm e V / 2)^{2}-\Delta_{S}^{2}}}, \quad F_{S}=\frac{\Delta_{S}}{\sqrt{\Delta_{S}^{2}-E^{2}}} .
$$

As can be seen from here, also using Sec. II D, the kinetic equations in the quasistationary limit coincide with the known adiabatic equations in the mixed representation as derived by Larkin and Ovchinnikov. ${ }^{38}$ In the absence of inelastic scattering, the equations further simplify to

$$
\begin{aligned}
& D_{T}(E) \frac{d^{2} f_{T}}{d x^{2}}(E, x)+\operatorname{Im} I_{S}(E) \frac{d f_{L}}{d x}(E, x)=0, \\
& D_{L}(E) \frac{d^{2} f_{L}}{d x^{2}}(E, x)+\operatorname{Im} I_{S}(E) \frac{d f_{T}}{d x}(E, x)=0 .
\end{aligned}
$$

Therefore, Eqs. (33) and (36) provide a set of equations to describe the time-dependent transport in double-barrier junctions in the limit of a large suppression parameter and a small voltage.

\section{B. Dissipative current}

From the expression for the dissipative current component in Eq. (23), and by solving Eqs. (33) and (36) with an ansatz $f_{T, L}=a_{1,2} x^{2}+b_{1,2} x+c_{1,2}$, it is obtained that

$$
I_{q p}(t)=\frac{1}{e R_{N}} \int_{-\infty}^{\infty} \frac{d E}{2} \frac{\gamma_{B 1}+\gamma_{B 2}}{\frac{\gamma_{B 1}}{M_{T 1}}+\frac{\gamma_{B 2}}{M_{T 2}}} f_{T 0} .
$$

This solution is obtained in the limit of no inelastic scattering. The effects of energy relaxation in the interlayer will be discussed in Sec. IV B. It follows from Eq. (37) that the quasiparticle current has in general a phase-dependent contribution through the coefficients $M_{T 1,2}$. The current is therefore time dependent since the phase difference is given by $\varphi=2 \mathrm{eVt} / \hbar$. The dc component is then determined by averaging over time.

When one of the superconducting electrodes is replaced by a normal metal, the expressions for $M_{T, L}$ simplify, since $F_{N}=0$. By putting voltage to zero in the superconductor, it can be shown that the expression for the quasiparticle current, Eq. (37) with $M_{T 2}=\operatorname{Re} G$, coincides with the known results of the SININ junction of Volkov et al. ${ }^{29}$ The additional term $m(E)=d^{-1} \int d x / M_{T}(E, x)$ in Ref. 29 is neglected in our case, since the term is small as compared to $\gamma_{B} / M_{T}$.

As a measure of the subgap conductance of a doublebarrier Josephson junction, the conductance at $e V=\Delta_{S}$ is calculated and shown in Fig. 4 as a function of temperature in the limit of $\gamma_{\text {eff }} \gg 1$. The inset of Fig. 4 shows the conductance at $e V=\Delta_{S}$ as a function of the inverse suppression parameter. It can be seen that the conductance is enhanced by a decrease in $\gamma_{\text {eff }}$. Physically, this corresponds to the opening of Andreev channels due to the term $\operatorname{Re} F_{S} \operatorname{Re} F$ in $M_{T}$. For $\gamma_{\text {eff }}>10$, for which the model of this section is a good approximation, the conductance is found to be approximately proportional to $\gamma_{\text {eff }}^{-1}$. This proportionality will be used in Sec. V to predict an intrinsic shunt in high- $J_{c}$ double-barrier Josephson junctions.

High voltage bias. In the regime of a large voltage $e V$ $\gg \Delta_{S}$, the time dependencies in the electrodes become decoupled and the current in an SINIS junction can be seen as the summation of the current in an SININ' junction and an N'INIS junction. In this case, the relevant functions $M_{T}$ in 


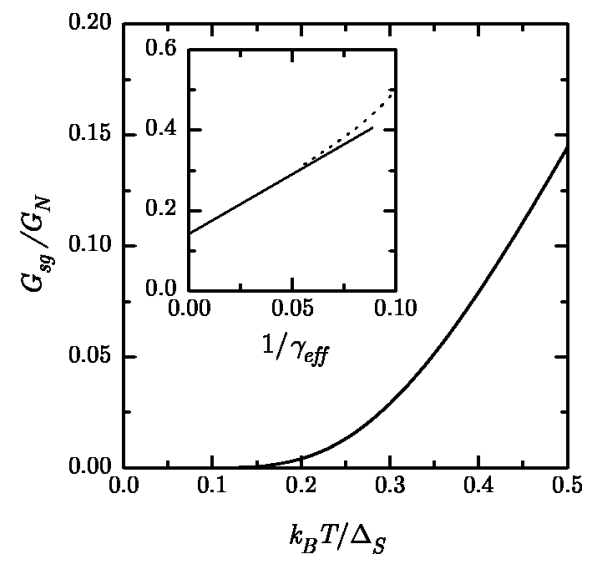

FIG. 4. Normalized conductance at $e V=\Delta_{S}$ as a function of temperature (normalized to $\Delta_{S}$ ) for $\gamma_{\mathrm{eff}} \gg 1$. The inset shows the normalized conductance at $e V=\Delta_{S}$ as a function of $1 / \gamma_{\mathrm{eff}}$ at $k_{B} T / \Delta_{S}=0.5$.

Eq. (37) simplify, and the presence of excess and deficit current can be calculated. Figure 5 shows the resulting dependence of the excess and deficit current on the asymmetry parameter, for several values of the suppression parameter. Note that the decoupling into SININ' and N'INIS junctions, at $e V \gg \Delta_{S}$ is valid for all values of $\gamma_{\mathrm{eff}}$. The limiting case of a deficit current $e I_{d e f} R_{N}=4 \Delta_{S} / 3$ for the symmetric limit and $\gamma_{\mathrm{eff}} \gg 1$ coincides with the findings of Zaitsev ${ }^{41}$ and Volkov et $a l .{ }^{29}$ The excess current $e I_{e x} R_{N} \simeq 1.05 \Delta_{S}$ for $\gamma_{\mathrm{eff}} \ll 1$ coincides with the results of MAR calculations. ${ }^{22}$

\section{Nonequilibrium supercurrent at finite voltage}

From Eq. (23) and a solution of the kinetic equations, the supercurrent can be determined as a function of voltage. In most tunnel junctions and weak links, the time dependence of the spectral supercurrent is harmonic and, by averaging over time, the supercurrent becomes zero at a finite voltage. However, due to the additional time dependence of $f_{L}$, the product of $f_{L}$ and $\operatorname{Im} I_{S}$ does not necessarily have to be harmonic, and a nonzero time-averaged supercurrent can exist at a finite voltage. Physically, the time-dependence of $f_{L}$ originates from the fact that, due to the proximity effect, the heat

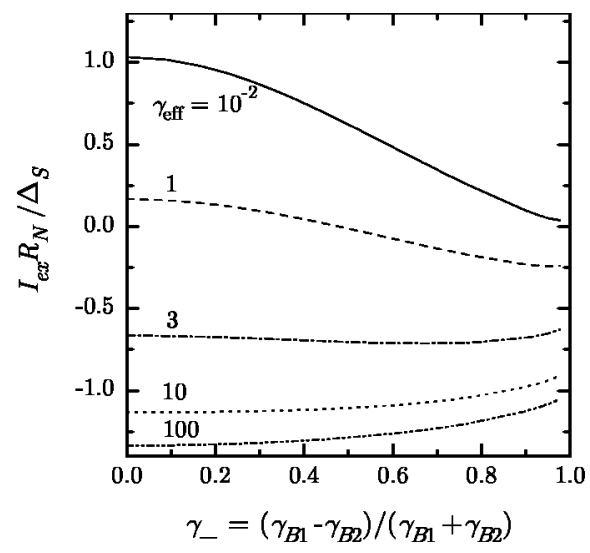

FIG. 5. Excess and deficit current as a function of asymmetry for several values of the suppression parameter. diffusion coefficient depends on the phase difference, as is known, e.g., from Andreev interferometers. ${ }^{49}$ We would like to calculate

$$
I_{S}(V)=\frac{1}{2 e R_{N}}\left\langle\int f_{L}(E, t) \operatorname{Im} I_{S}(E, t) d E\right\rangle_{t},
$$

where the brackets denote time averaging. It can be seen that this expression explicitly depends on the spectral supercurrent, which could, for example, be suppressed by a magnetic field. Therefore, this current contribution is true supercurrent in the presence of an applied voltage.

Since the time-dependent perturbation of $f_{L}$ is much smaller than $f_{0}$, kinetic equations $(21)$ can be rewritten in the adiabatic form like Eqs. (36), now including inelastic scattering,

$$
\begin{gathered}
D_{T} \frac{d^{2} f_{T}}{d x^{2}}+\operatorname{Im} I_{S} \frac{d f_{L}}{d x}=\delta^{-1} f_{T}, \\
D_{L} \frac{d^{2} f_{L}}{d x^{2}}+\operatorname{Im} I_{S} \frac{d f_{T}}{d x}=\delta^{-1}\left(f_{L}-f_{0}\right),
\end{gathered}
$$

where, $\delta$ is introduced as $\delta^{-1}=N \xi^{2} / \mathcal{D} \tau_{\text {in }}$. Under the conditions of the adiabatic approximation, we can use again boundary conditions (33), and, with ansatz solutions $f_{T}$ $=a_{1} x^{2}+b_{1} x+c_{1}$ and $f_{L}=a_{2} x^{2}+b_{2} x+c_{2}$, this provides us with the solution

$$
f_{L}-f_{0}=\gamma_{\mathrm{eff}} \operatorname{Im} I_{S} \frac{M}{\gamma_{B} D_{T}} f_{T 0},
$$

where

$$
M=\frac{2 M_{T 1} M_{T 2}+2 \gamma_{\mathrm{eff}} \delta^{-1} M_{T 1}}{\left(M_{T 1}+M_{T 2}+2 \gamma_{\mathrm{eff}} \delta^{-1}\right)\left(M_{L 1}+M_{L 2}+2 \gamma_{\mathrm{eff}} \delta^{-1}\right)} .
$$

From Eqs. (40) and (41) it can be seen that in the limit of strong inelastic scattering, $\tau_{i n} \rightarrow 0, f_{L}-f_{0}$ will be proportional to $\tau_{i n}$ and therefore equilibrium is restored.

The nonequilibrium correction to $f_{L}$ is obtained within the adiabatic approximation. Therefore, we assume that time dependence only comes into the final expressions via the phase factor in the spectral supercurrent density $\operatorname{Im} I_{S}$. In the case of symmetric barriers, $\operatorname{Im}^{2} I_{S}$ averaged over time is equal to the average of sine-squared, which is just a factor $1 / 2$. The supercurrent contribution can therefore be written as

$$
I_{S}=\frac{1}{2 e R_{N}} \gamma_{\mathrm{eff}} \int_{0}^{\infty} D_{T}^{-1}\left(\operatorname{Im} I_{S}\right)^{2} f_{T 0} M d E
$$

In order to perform the integral some smearing of the $\operatorname{Im} I_{S}^{2}$ divergency has to be assumed. Physical reasons for this are always present, like a small amount of inelastic scattering. An inelastic scattering term $\gamma$ can be taken into account in the retarded part of the Usadel equations, ${ }^{35}$ but in the limit of little inelastic scattering, $\gamma \ll k_{B} T_{c S}$, the scattering term can be presented in the solutions by transforming the energy $E$ to 


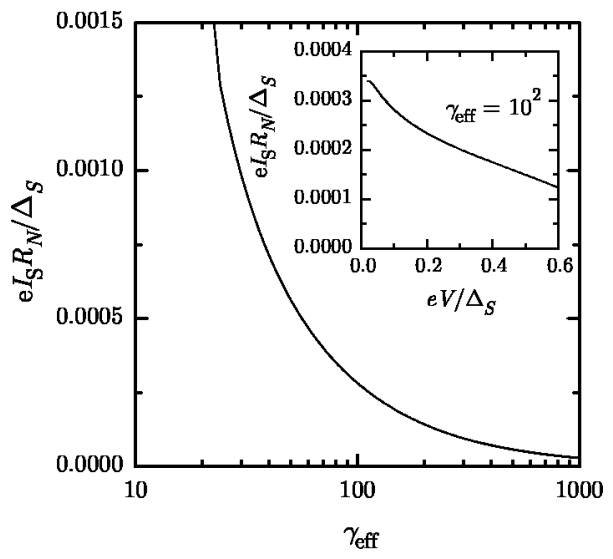

FIG. 6. Nonequilibrium averaged supercurrent at a finite voltage bias $\left(e V=0.1 \Delta_{S}\right)$ at $k_{B} T=0.2 \Delta_{S}$ and a small inelastic scattering rate $\gamma=10^{-3} \Delta_{S}$ as a function of the suppression parameter. The inset shows the supercurrent as a function of bias voltage at $k_{B} T$ $=0.2 \Delta_{S}$ for a fixed suppression parameter $\gamma_{\mathrm{eff}}$.

$E+i \gamma$. In Sec. V, realistic values of the inelastic scattering parameter and the suppression parameter will be derived. From these values it can be concluded that $\gamma_{\mathrm{eff}} \delta^{-1} \ll 1$ in most of the practical cases. In this limit, the only contribution to the supercurrent comes from $E>\Delta_{S}$, the spectral supercurrent being zero below the minigap and $M$ being zero between the minigap and $\Delta_{S}$. The resulting supercurrent in this limit is shown in Fig. 6 as a function of the suppression parameter and in the inset as a function of voltage.

The physics of this effect is similar to the physics of the nonequilibrium supercurrent first considered by Lempitskii ${ }^{31}$ for a long diffusive SNS junction without potential barriers at the NS interfaces. The mechanism is the conversion of quasiparticle current into supercurrent inside the junction, formally described by the coupling term $\operatorname{Im} I_{S} d f_{T} / d x$ in Eq. (39). An alternative explanation for this mechanism has been given in terms of thermoelectricity in Ref. 49. However, quantitative differences occur between the cases of long and short junctions. In a long SNS junction with $d \gg \xi$ the strongest deviations of the distribution function $f_{L}$ from equilibrium occur at the subgap energy range, at energies of the order of the Thouless energy $\hbar \mathcal{D} / d^{2}$. In the case of MAR, an additional nonequilibrium correction to $f_{L}$ appears, as shown by Pierre et al., ${ }^{18}$ which is beyond the present adiabatic approach in which voltage and interface transparencies are small so that the MAR is suppressed. At subgap energies the excitation of the symmetric mode described by $f_{L}$, generated by the quasiparticle current, cannot diffuse out of the junction since the corresponding diffusion constant $D_{L}$ vanishes in the $\mathrm{S}$ electrodes. On the other hand, in SINIS junctions, the symmetric mode at low bias is excited only at $E>\Delta_{S}$ since the quasiparticle current vanishes in the energy range $E<\Delta_{S}$ due to the presence of tunnel barriers. Hence, deviations of $f_{L}$ from equilibrium occur only at $E>\Delta_{S}$. Since the diffusion constant in the $\mathrm{S}$ electrodes $D_{L}<1$ at $E>\Delta_{S}$, the excitations at $E>\Delta_{S}$ are partially trapped at these energies, though the magnitude of the effect in SINIS junctions is smaller than in SNS junctions. With a decreasing barrier height in SINIS junctions, an Andreev contribution to the quasiparticle current appears and deviations from equilibrium should also occur at $E<\Delta_{S}$. The study of this crossover, however, is beyond the scope of this paper.

The nonequilibrium supercurrent at a certain voltage is approximately two order of magnitude smaller than the dc supercurrent at zero voltage, but is comparable with the quasiparticle current at the same voltage. The latter conclusion is based on the assumption that inelastic scattering is negligible, but we will see in Sec. IV that energy relaxation in the interlayer increases the subgap quasiparticle current. Therefore we can conclude that the nonequilibrium supercurrent can be present in double-barrier junctions, but that under realistic junction parameter values, the contribution to the total current is minor. Note, that the nonequilibrium supercurrent in the considered regime is proportional to the square of the sine of the phase difference over the junction, $I_{S}$ $\sim \sin ^{2} \phi$. This effect can give rise to the occurence of halfinteger Shapiro steps in the $I V$ characteristics when the junction is irradiated by microwaves. ${ }^{50,51}$

\section{INELASTIC SCATTERING}

In many mesoscopic systems and weak links, the time of flight of a quasiparticle through a normal metal or superconducting layer is much shorter than the characteristic inelastic scattering time in the specific material. Hence, inelastic scattering in mesoscopic systems and weak links is usually neglected. However, in double-barrier junctions, the time of flight can be large. Because of the normal reflections at the interfaces, a quasiparticle on average traverses the interlayer many times. The time that a quasiparticle effectively spends in the interlayer is proportional to $D^{-1}$, where $D$ is the transparency of each barrier. For a transparency of the order of $10^{-6}$, the time of flight in the interlayer is for example of the order of $\tau=d / D v_{F}=0.5 \mathrm{~ns}$, for a thickness of about $10 \mathrm{~nm}$ and a typical Fermi velocity of $1.5 \times 10^{6} \mathrm{~m} / \mathrm{s} .^{52}$

In most double-barrier Josephson junctions $\mathrm{Al}$ is used as an interlayer material, and therefore the inelastic scattering time in $\mathrm{Al}$ should be considered. Kaplan et al. ${ }^{53}$ estimated an inelastic scattering time in bulk $\mathrm{Al}$ of $400 \mathrm{~ns}$ which is much larger than $0.5 \mathrm{~ns}$. However, magnetoresistance and microwave measurements in thin films of Al (Refs. 54 and 55) showed that the inelastic scattering time in thin $\mathrm{Al}$ films is orders of magnitudes smaller than in bulk, namely, of the order of 0.1 to $1.0 \mathrm{~ns}$ in films of a few to 10-nm thickness. Therefore, in the modeling of time-dependent transport properties of double-barrier junctions, inelastic scattering, or energy relaxation, has to be taken into account. The inelastic scattering comprises both electron-phonon and electronelectron scattering.

\section{A. Derivation of a microscopic model}

In this section, a microscopic model will be derived for the quasiparticle current as function of voltage in doublebarrier Josephson junctions with low-transparent barriers. It will be shown that the results coincide with the phenomenological model by Heslinga and Klapwijk ${ }^{56}$ who derived their model by matching the population and extraction rates of the quasiparticles in the interlayer. 
In this section, the assumption will be made that $\gamma_{\mathrm{eff}}$ $\gg 1$. In this approximation, the proximity effect can be neglected, i.e. $F^{R(A)}=0$. Furthermore, the spectral supercurrent $\operatorname{Im} I_{S}(E, t)=0, \operatorname{Re} G(E)=1$, and $D_{L}=D_{T}=1$. In this case, none of the quantities explicitly depends on time. Then, the kinetic Eqs. (20) can be simplified to

$$
\begin{gathered}
\mathcal{D} \tau_{i n} \frac{\partial^{2}}{\partial x^{2}} f_{L}(E, x)-\left[f_{L}(E, x)-f_{0}(E)\right]=0, \\
\mathcal{D} \tau_{i n} \frac{\partial^{2}}{\partial x^{2}} f_{T}(E, x)-f_{T}(E, x)=0
\end{gathered}
$$

where $f_{0}(E)=\tanh (E / 2 T)$ and $\mathcal{D}$ is the diffusion constant in the interlayer. Use is made of the fact that $f_{T 0}(E)=0$ in equilibrium. The kinetic equations are decoupled in this case, but $f_{T}$ and $f_{L}$ are coupled through the boundary conditions.

The boundary conditions can either be obtained by simplifying the relevant terms of the expressions that contain all harmonics, such as Eqs. (30) and (31), or by starting from the time-dependent boundary conditions, [Eqs. (28) and (29)]. In the latter case, the transformation to energy space is straightforward. The right-hand sides of Eqs. (28) and (29) only contain terms that depend on time difference since $F^{R(A)}=0$, e.g.,

$$
\begin{gathered}
\operatorname{Re} G_{1}\left(t-t_{1}\right) \circ i \sin \frac{e V\left(t-t_{1}\right)}{\hbar} \circ \operatorname{Re} G\left(t_{1}-t^{\prime}\right) \circ f_{L}\left(t_{1}-t^{\prime}\right) \\
=\int d E e^{-i E\left(t-t^{\prime}\right) / \hbar} \operatorname{Re} G(E) f_{L}(E) G_{-},
\end{gathered}
$$

where $G_{-}=\operatorname{Re} G_{1}(E+e V / 2)+\operatorname{Re} G_{1}(E-e V / 2)$. The lefthand side of Eq. (28) becomes

$$
D_{T} \gamma_{B} \xi \frac{\partial}{\partial x} f_{T}\left(t, t^{\prime}\right)=\gamma_{B} \xi \frac{\partial}{\partial x} \int d E e^{-i E\left(t-t^{\prime}\right)} D_{T}(E) f_{T}(E)
$$

Hence, together with $\operatorname{Re} G=1$, finally the boundary conditions read

$$
\begin{aligned}
\gamma_{B} \xi \frac{\partial}{\partial x} f_{T}(E, \pm d / 2)= & \mp f_{T}(E, \pm d / 2) N_{+} \\
& -f_{L}(E, \pm d / 2) N_{-}+R_{-}, \\
\gamma_{B} \xi \frac{\partial}{\partial x} f_{L}(E, \pm d / 2)= & \mp f_{L}(E, \pm d / 2) N_{+} \\
& -f_{T}(E, \pm d / 2) N_{-} \pm R_{-},
\end{aligned}
$$

where $N_{ \pm}=\operatorname{Re} G_{1}(E+e V / 2) \pm \operatorname{Re} G_{1}(E-e V / 2)$ in the superconductors and $R_{ \pm}=\operatorname{Re} G_{1}(E+e V / 2) \times f_{0}(E+e V / 2)$ $\pm \operatorname{Re} G_{1}(E-e V / 2) f_{0}(E-e V / 2)$. The kinetic equations provide that $c_{1}=2 a_{1} D \tau_{i n}=2 a_{1} \delta$ for the ansatz $f_{T}=a_{1} x^{2}$ $+b_{1} x+c_{1}$ and $f_{L}=a_{2} x^{2}+b_{2} x+c_{2}$. Using boundary conditions (46) and neglecting terms proportional to $d^{2}$, the solution can be simply found. The quasiparticle current is given by Eq. (23), where $d f_{T} / d x=b_{1}$, and $b_{1}$ is given by

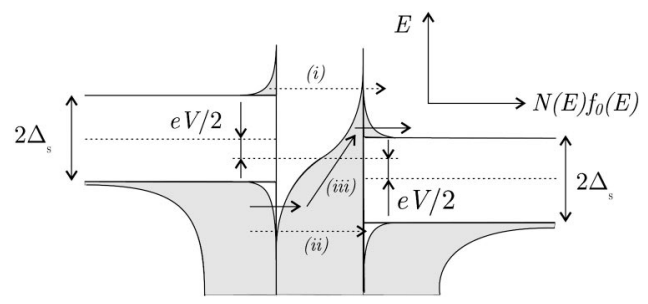

FIG. 7. Semiconductor-diagram representation of tunnel and scattering rates in a double-barrier junction at bias voltage $\mathrm{eV}$ $=\Delta_{S}$. The energy conserving processes (i) and (ii) are, in the case of inelastic scattering, complemented by process (iii).

$$
b_{1}=\frac{1}{\gamma_{B} \xi} \frac{R_{-} N_{+}-R_{+} N_{-}+\left(R_{-}-f_{0} N_{-}\right) / \Gamma \tau_{i n}}{N_{+}+1 / \Gamma \tau_{\text {in }}},
$$

where $\Gamma^{-1}=\gamma_{B} d \xi / \mathcal{D} \hbar=e^{2} N(0) R_{B} d / \hbar$ is the tunneling injection rate into the normal metal interlayer, $N(0)$ the unnormalized density of states in the interlayer and $R_{B}$ the specific barrier resistance. Using the fact that density of states functions are symmetric in energy and $f_{0}$ is asymmetric in energy, $\left(R_{-}-f_{0} N_{-}\right)$can be simplified to $2 \operatorname{Re} G_{1}(E$ $+e V / 2)\left[f_{0}(E+e V / 2)-f_{0}(E)\right] \quad$ and $\quad R_{-} N_{+}-R_{+} N_{-}$ $=2 \operatorname{Re} G_{1}(E+e V / 2) \operatorname{Re} G_{1}(E-e V / 2)\left[f_{0}(E+e V / 2)-f_{0}((E\right.$ $-e V / 2))]$. With $\sigma_{N} / 2 \gamma_{B}=e^{2} N(0) \mathcal{D} / \gamma_{B}=R_{B}^{-1}$, the expression for the quasiparticle current finally becomes

$$
\begin{aligned}
I= & \frac{2}{e R_{N}} \int_{-\infty}^{\infty} d E \operatorname{Re} G_{1}(E+e V / 2) \\
& \times \frac{\operatorname{Re} G_{1}\left(E-\frac{e V}{2}\right) F_{-}+\left[f_{0}\left(E+\frac{e V}{2}\right)-f_{0}(E)\right] / \Gamma \tau_{\text {in }}}{G_{+}+1 / \Gamma \tau_{\text {in }}},
\end{aligned}
$$

where $F=f_{0}(E+e V / 2)-f_{0}(E-e V / 2)$ and $G_{+}=\operatorname{Re} G_{1}(E$ $+e V / 2)+\operatorname{Re} G_{1}(E-e V / 2)$. This expression is equivalent to the findings of Heslinga and Klapwijk, ${ }^{56}$ in the limit of $\operatorname{Re} G=1$, who derived a model by equating the population and extraction rates in the interlayer. Zaitsev's results for the SININ junction in the limit of no energy relaxation ${ }^{28}$ coincide with our findings as well. The equivalence of a mesoscopic or phenomenological approach and the more rigorous Green's functions treatment is shown by Argaman ${ }^{50}$ to hold for the equations for current. Here we have proven that the final expression [Eq. (48)] also follows from the Green's function approach, using the appropriate boundary conditions.

\section{B. Influence of inelastic scattering on transport properties}

Examples of possible tunneling processes are indicated in Fig. 7. In one of the processes a quasiparticle is inelastically scattered in the interlayer. Equation (48) coincides in the limit of strong inelastic scattering $\left(\Gamma \tau_{i n}=0\right)$ with the known result for two SIN tunnel junctions in series. In the absence of inelastic scattering, Eq. (48) reduces to 


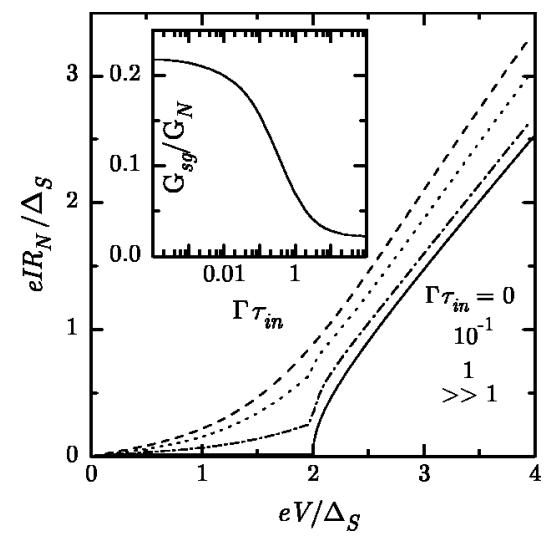

FIG. 8. $I V$ characteristics at $k_{B} T / \Delta_{S}=0.25$ and $\gamma_{\text {eff }} \gg 1$ on the basis of Eq. (48) for several values of the inelastic scattering parameter $\Gamma \tau_{i n}$. The inset shows the subgap conductance at $e V=\Delta$ as a function of $\Gamma \tau_{i n}$.

$$
I=\int_{-\infty}^{\infty} \frac{2 d E}{e R_{N}} \operatorname{Re} G_{1}\left(E+\frac{e V}{2}\right) \operatorname{Re} G_{1}\left(E-\frac{e V}{2}\right) \frac{F_{-}}{G_{+}} .
$$

Figure 8 shows both limiting cases as well as $I V$ curves for intermediate values of the scattering parameter, taken in the present limit of $\gamma_{\mathrm{eff}} \gg 1$. It can be seen in the inset of Fig. 8, that inelastic scattering enhances the subgap conductance. This effect will be discussed in section $\mathrm{V}$ in order to explain the large subgap conductance observed in double-barrier junction measurements.

Equation (49) gives a deficit current of $e I_{d e f} R_{N}=4 \Delta_{S} / 3$ for $e V \gg \Delta_{S}$, which coincides with the findings of Sec. III B. In analogy with the approach of Sec. III B to calculate excess and deficit currents by summing the respective contributions from SININ and NINIS junctions, the same can be calculated by including inelastic scattering as well. Figure 9 shows the resulting crossover from excess to deficit current as function of the suppression parameter for several values of the inelastic scattering parameter. For $\Gamma \tau_{i n}<10^{-1}$ only a small deficit current is predicted at $e V \gg \Delta_{S}$. However, at moderate values of $V$, i.e., $2 \Delta_{S}<e V<4 \Delta_{S}$, a considerable deficit current is still present, as can be seen, for example, in Fig. 8.

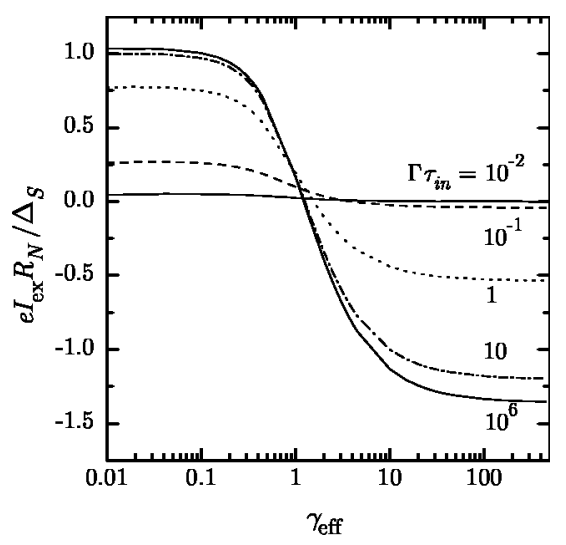

FIG. 9. Excess and deficit current as function of the suppression parameter for several values of the inelastic scattering parameter $\Gamma \tau_{\text {in }}$.

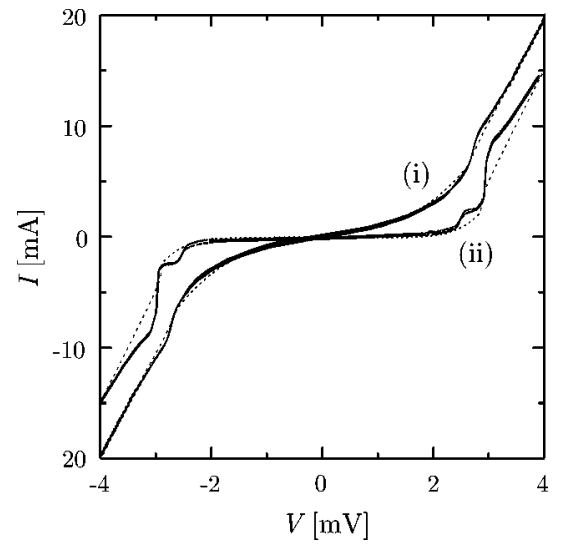

FIG. 10. Experimental $I V$ curves (solid lines) at $4.2 \mathrm{~K}$ (i) and $1.6 \mathrm{~K}$ (ii) together with theoretical fits (dashed lines) with $\Gamma \tau_{\text {in }}$ $=0.1$ and 0.3 , respectively.

\section{APPLICATION: THE NATURE OF THE INTRINSIC SHUNT}

The resistively and capacitively shunted junction model shows how a sinusoidal supercurrent-phase relation, a linear quasiparticle current, and a displacement current determine the shape of the entire $I V$ characteristic of a Josephson junction. The model can also be applied to an unshunted junction, but then the subgap resistance $R_{s g}$ appears in the expression for the Stewart-McCumber parameter

$$
\beta_{c}=2 \pi \frac{\left(I_{c} R_{N}\right)^{2} C}{I_{c} \Phi_{0}}\left(\frac{R_{s g}}{R_{N}}\right)^{2},
$$

where $C$ is the capacitance of the junction and $\Phi_{0}(=2.07$ $\left.\times 10^{-15} \mathrm{~Wb}\right)$ the flux quantum. Likharev ${ }^{57}$ showed that the relation between $\beta_{C}$ and the presence of hysteresis depends on the model that is used to describe the junction (e.g., the nonlinear resistive model, with different dependencies for the subgap conductance, and the tunnel junction microscopic model), but roughly speaking, it can be said that $\beta_{C}>1$ corresponds to hysteretic $I V$ characteristics. Hysteresis refers here to the existence of two branches in the $I V$ curve, one going from the critical current $I_{c}$ to the voltage state, and one going back at the return current $\left(I_{R}<I_{c}\right)$ from the voltage state to the state at $V=0$.

The capacitance of a double-barrier junction is not known a priori. In Ref. 58 a set of $\mathrm{Nb} / \mathrm{Al}$ double-barrier Josephson junctions was fabricated in order to make superconducting quantum interference devices (SQUIDs). From resonances in the SQUID washer, $C$ was determined to be $0.015 \mathrm{pF} / \mu \mathrm{m}^{2}$, corresponding to the capacitance of two SIS junctions in series. ${ }^{58}$ It is assumed that this value is only weakly depending on the transparency of the barrier. The dependence of $I_{c}$ and $R_{N}$ on the junction parameters, such as $\gamma_{\text {eff }}$, follow from the modeling of the stationary properties in Ref. 26. The subgap conductance as function of the suppression parameter is determined in Sec. III B.

First, the regime of junctions with $\gamma_{\mathrm{eff}} \gg 1$ will be discussed. For this purpose, low critical current density $\mathrm{Nb} / \mathrm{Al}$ double-barrier junctions were fabricated according to the process of Ref. 58. Figure 10 shows a typical measured $I V$ 
characteristic, together with an $I V$ curve from the nonequilibrium model of Sec. IV B, where inelastic scattering is taken into account. At $4.2 \mathrm{~K}$, the experimental and theoretical curves are very much alike, taking into account the fact that only one free parameter was used to fit, namely, $\Gamma \tau_{\text {in }}$. From

$$
\Gamma \tau_{i n}=\frac{\pi T_{c S} k_{B}}{\gamma_{\mathrm{eff}}} \frac{\tau_{i n}}{h},
$$

and the fitted $\Gamma \tau_{i n}=0.1$ and $\gamma_{\text {eff }}=2 \times 10^{3}$ (which was obtained from fitting the critical current temperature dependence), an inelastic scattering time $\tau_{i n}=0.3 \mathrm{~ns}$ is obtained in the Al interlayers. At $1.6 \mathrm{~K}$, a magnetic field was used to suppress the supercurrent in order to resolve the subgap quasiparticle conductance. The deviation of the fit from the experiment around $2 \Delta_{N b}$ is due to the nonequilibrium enhancement of the gap in the interlayer, as described in Ref. 59, which can be included in the model by incorporating $\Delta_{A l}$. However, the good fit well below $2 \Delta_{N b}$ allows for the extraction of $\tau_{i n}=0.9 \mathrm{~ns}$ at $1.6 \mathrm{~K}$.

The values for inelastic scattering correspond to measurements by Santanam et al., ${ }^{55}$ who found $\tau_{\text {in }}=0.2-1.0 \mathrm{~ns}$ in 10 -nm Al films at $4.2 \mathrm{~K}$, and Van Son et al. ${ }^{54}$ who found $\tau_{i n}=0.8-0.9 \mathrm{~ns}$ in $7-\mathrm{nm} \mathrm{Al}$ films at $T_{c A l}$. Our values of $\tau_{i n}=0.9 \mathrm{~ns}$ at $1.6 \mathrm{~K}$ and $0.3 \mathrm{~ns}$ at $4.2 \mathrm{~K}$ indicate a scaling with $T^{-1}$ rather than $T^{-3}$, which was found and discussed as well by Santanam et al. ${ }^{55}$ Note that the values for the inelastic scattering are much smaller than $\pi k_{B} T$, which means that the stationary properties are not influenced by $\tau_{\text {in }}$. Furthermore, from these values it is seen that $\gamma_{\mathrm{eff}} \delta^{-1} \gg 1$ as long as $\gamma_{\text {eff }} \leqslant 10^{3}$, which was used in order to obtain Fig. 6 .

As a measure of the subgap conductance, the theoretically expected normalized conductance at $e V=\Delta_{S}$ can be found in Fig. 8, as function of the inelastic scattering parameter $\Gamma \tau_{i n}$. It can be seen that the subgap resistance in the limit of zero inelastic scattering $\left(\tau_{i n} \rightarrow \infty\right)$ is only determined by the temperature. The conductance in this limit is therefore called the thermal contribution. The relation between subgap resistance and $\gamma_{\text {eff }}$ is now known for a fixed value of $\tau_{\text {in }}$ since $\Gamma$ is given by $\pi k_{B} T / \gamma_{\mathrm{eff}}$.

The dependence of $I_{c} R_{N}$ on $\gamma_{\mathrm{eff}}$ is known from the Matsubara modeling of the stationary properties of doublebarrier junctions. ${ }^{26}$ Together with the definition of $\gamma_{\text {eff }}$, it follows that

$$
R_{N}^{-1}=\frac{e^{2} k_{F}^{2}}{2 \pi^{2} \hbar} \frac{\pi k_{B} T_{c S} d}{\hbar v_{F} \gamma_{\mathrm{eff}}}
$$

where the parameter values can be taken as $v_{F}=1.5 \times 10^{6}$ $\mathrm{m} / \mathrm{s},{ }^{52} d=6 \mathrm{~nm}$, and $T_{c N b}=9.2 \mathrm{~K}$. Putting these theoretical dependencies together with the experimentally determined parameters into Eq. (50), provides $\beta_{C}$ as function of the critical current density for junctions with $\gamma_{\mathrm{eff}} \gg 1$, see Fig. 11 .

The shunting behavior can physically be explained as follows. A direct transfer process of quasiparticles from one electrode to the other is prohibited when the quasiparticle energy falls within the gap of the other electrode. However, by scattering inelastically in the interlayer, the quasiparticles

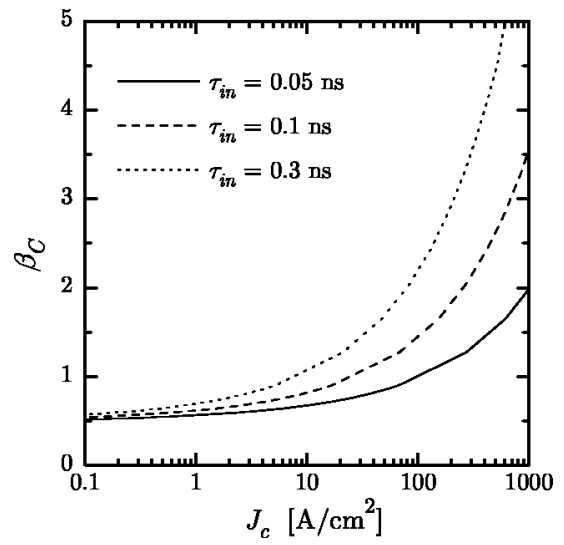

FIG. 11. Expected $\beta_{C}$ as a function of critical current density from the inelastic scattering model, $T=4.2 \mathrm{~K}$.

are redistributed over energy, allowing some quasiparticles to enter the other electrode, which results in an enhanced conductance, as illustrated in Fig. 7. The amount of quasiparticles that is scattered inelastically increases for decreasing barrier transparencies, since the effective lifetime of a quasiparticle in the interlayer is then increased. For strong inelastic scattering, the double-barrier junction can be regarded as a series connection of SIN and NIS junctions, where the energy distibution function in the interlayer is the equilibrium Fermi function $f_{0}=\tanh \left(E / 2 k_{B} T\right)$. Here it should be noted that the assumption is made that the inelastic scattering is dominated by electron-phonon interactions, and that there is coupling between the interlayer and a heat bath. It is known $^{18}$ that, in the contrary nonadiabatic limit of MAR and strong electron-electron interactions, the energy distribution in the interlayer is given by the Fermi function at a temperature $k_{B} T=\Delta+e V$.

In order to understand the intrinsic shunt of all Al-based double-barrier junctions, the regime of high- $J_{c}$ junctions (typically larger than $100 \mathrm{~A} / \mathrm{cm}^{2}$ ) should be considered as well. The second contribution to the subgap conductance is due to the Andreev reflection processes at the two superconductor-normal metal interfaces, which was formally introduced by the term $\operatorname{Re}(F) \operatorname{Re}\left(F_{S}\right)$. The Andreev channels open at a high transparency of the interface barriers. In first order, this contribution is independent of temperature, but it depends on the suppression parameter, which is shown in the inset of Fig. 4 for a fixed temperature. For the practical range of parameters, this means that the contribution is inversely proportional to $\gamma_{\mathrm{eff}}$. Figure 12 shows the resulting hysteresis as function of the critical current density. Figure 12 predicts that nonhysteretic double-barrier junctions can be obtained with critical current densities of the order of $10 \mathrm{kA} / \mathrm{cm}^{2}$ and higher. In order to make a comparison with SIS junctions, a similar curve has been calculated based on Eq. (50) and plotted in Fig. 13. In this calculation it was assumed that $C$ $=3.0 \mu \mathrm{F} / \mathrm{cm}^{2}, I_{c} R_{N}=2.0 \mathrm{mV}$, and $R_{s g}=2 R_{N}$. A bigger subgap resistance will shift the SIS curve even more to the right.

Summing up all contributions to the subgap conductance provides the theoretical curve in Fig. 13 for several values of $\tau_{\text {in }}$ and $d=6 \mathrm{~nm}$, where Zappe's equation ${ }^{60}$ was used to calculate the ratio of return and critical current $I_{R} / I_{c}$ from $\beta_{C}$. The summation is performed in a straightforward way, since the contributions due to inelastic scattering and the 


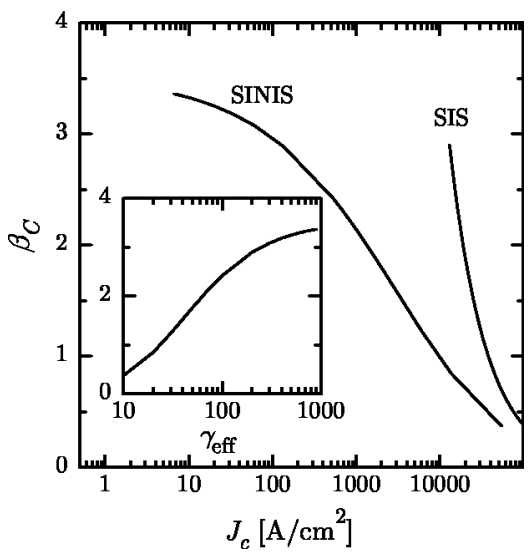

FIG. 12. Theoretical model for $\beta_{C}$ as function of $J_{c}$ (and as function of $\gamma_{\mathrm{eff}}$ in the inset), based on the contribution of Andreev channels to the subgap conductance in high- $J_{c}$ junctions at $T=4.2$ $\mathrm{K}$, in comparison with the hysteresis of SIS junctions.

opening of Andreev channels do not overlap, i.e., they occur in separate regimes of the suppression parameter.

An increase in $\tau_{\text {in }}$ is seen to increase the hysteresis and shift the maximum hysteresis to lower values of $J_{c}$. A thicker interlayer will both decrease $J_{c}$ as well as shift the curve upward, since $\Gamma \tau_{\text {in }}$ is larger in this case. A decrease in temperature rapidly enhances the hysteresis, since both the thermal contribution to the subgap conductance decreases as well as the contribution of inelastic scattering, since $\tau_{\text {in }}$ increases with temperature. This explains the strong influence of temperature on hysteresis as observed in experiments, which is stronger than could be expected from an increase in $I_{c}$ alone.

Observed experimental $I_{R} / I_{c}$ values are also shown in Fig. 13, and it can be concluded that the experiments are now qualitatively and quantitatively very well explained by the model in the sense that both the nonmonotonic hysteresis dependence on critical current density as well as the actual hysteresis values are obtained.

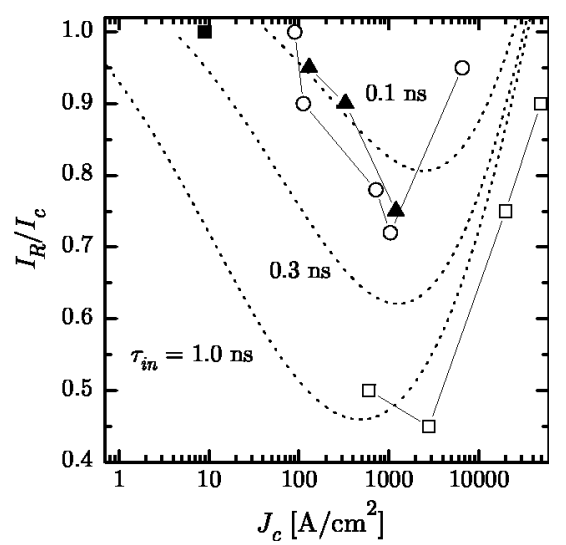

FIG. 13. Theoretical model (dotted line) for the ratio of return and critical current, based on the sum of the shunting contributions at $4.2 \mathrm{~K}$ from both inelastic scattering and Andreev channels, for several inelastic scattering times. Experimental data are shown from this paper $(\boldsymbol{\square})$, Ref. $61\left({ }^{\circ}\right)$, Ref. $62(\square)$, and Ref. $63(\boldsymbol{\Delta})$.

\section{CONCLUSION}

Time-dependent and nonequilibrium transport properties of SINIS junctions have been studied by means of a microscopic Green's function approach. The kinetic equations for the longitudinal and transverse energy distribution functions are derived from the Keldysh-Usadel equation. The appropriate boundary conditions are derived by starting from the Kupriyanov-Lukichev boundary conditions and by applying gauge transformations in the electrodes. The resulting set of equations has a recurrent nature, in terms of coupling of Green's functions to higher harmonics as well as to functions with shifted energy arguments. This lays out a theoretical framework to microscopically study time-dependent problems in superconducting - normal metal devices.

We apply this formalism in order to develop a theory of the subgap conductance of SINIS junctions. This conductance is a very favorable feature for applications but so far not understood on a microscopic level. In the adiabatic limit of a small voltage and a large suppression parameter, the time dependencies simplify and the equations are solved to determine the dissipative current in double-barrier Josephson junctions. Known limiting cases, such as the SININ' junction, are reproduced. Excess and deficit current are determined as function of the suppression parameter and the asymmetry between the barriers. Excess current as high as $e I_{e x} R_{N} \simeq 1.05 \Delta_{S}$ can exist in double-barrier junctions in the symmetric case for $\gamma_{\mathrm{eff}} \ll 1$, and maximum deficit current is reached in the symmetric case for $\gamma_{\text {eff }} \gg 1$. The subgap conductance enhancement by decreasing $\gamma_{\mathrm{eff}}$ is caused by the opening of Andreev channels.

It is found that the time-dependent nonequilibrium contribution to the energy distribution function gives rise to a nonzero averaged supercurrent in the presence of a voltage bias. This effect should be observable in double-barrier junction experiments.

In contrast to most studied mesoscopic systems, inelastic scattering in the interlayer of double-barrier junctions can have a strong influence on the electronic transport even in very short devices. A microscopic derivation of the dependence of the transport properties on the inelastic scattering parameter is given. $I V$ characteristics show an enhanced subgap conductance for increased inelastic scattering rates.

The actual value for the inelastic scattering time in the interlayer of experimentally realized devices was obtained by fitting the microscopic model. The inelastic scattering values explain, together with the opening of Andreev channels, the nature of the intrinsic shunt in double-barrier junctions.

\section{ACKNOWLEDGMENTS}

This project was supported by the Dutch Foundation for Fundamental Research on Matter (FOM). We thank H. Hilgenkamp, T.M. Klapwijk, Th. Schäpers, V.S. Shumeiko, and A.D. Zaikin for valuable discussions. F.K.W. is grateful to B. Pannetier and H. Courtois for hospitality and discussions during the very early stages of this project. 


\section{APPENDIX A: DERIVATION OF THE BOUNDARY CONDITIONS}

From the definition of the Green's functions in Keldysh $\times$ Nambu space [Eq. (1)], a boundary condition can be written for each of the matrix elements of Eq. (24):

$$
\begin{gathered}
\gamma_{B} \xi \hat{G}^{R} \frac{d}{d x} \hat{G}^{R}=\hat{G}^{R} \hat{G}_{1}^{R}-\hat{G}_{1}^{R} \hat{G}^{R}, \\
\gamma_{B} \xi \hat{G}^{A} \frac{d}{d x} \hat{G}^{A}=\hat{G}^{A} \hat{G}_{1}^{A}-\hat{G}_{1}^{A} \hat{G}^{A}, \\
\gamma_{B} \xi\left(\hat{G}^{R} \frac{d}{d x} \hat{G}^{K}+\hat{G}^{K} \frac{d}{d x} \hat{G}^{A}\right) \\
=\hat{G}^{R} \hat{G}_{1}^{K}+\hat{G}^{K} \hat{G}_{1}^{A}-\hat{G}_{1}^{R} \hat{G}^{K}-\hat{G}_{1}^{K} \hat{G}^{A} .
\end{gathered}
$$

With definition (18), the left-hand side of the latter of these three boundary conditions becomes

$$
\begin{aligned}
& \gamma_{B} \xi\left[\left(\hat{G}^{R} \frac{d}{d x} \hat{G}^{R}\right) \hat{f}+\hat{G}^{R} \hat{G}^{R} \frac{d}{d x} \hat{f}-\hat{G}^{R} \frac{d}{d x}\left(\hat{f} \hat{G}^{A}\right)\right. \\
& \left.+\hat{G}^{R} \hat{f} \frac{d}{d x} \hat{G}^{A}+\hat{f} \hat{G}^{A} \frac{d}{d x} \hat{G}^{A}\right] .
\end{aligned}
$$

With the aid of the first two equations in Eq. (A1) this can be rewritten into

$$
\begin{aligned}
\gamma_{B} \xi[ & \left.\hat{G}^{R} \hat{G}^{R} \frac{d}{d x} \hat{f}-\hat{G}^{R} \frac{d}{d x}\left(\hat{f} \hat{G}^{A}\right)+\hat{G}^{R} \hat{f} \frac{d}{d x} \hat{G}^{A}\right] \\
& +\left(\hat{G}^{R} \hat{G}_{1}^{R}-\hat{G}_{1}^{R} \hat{G}^{R}\right) \hat{f}-\hat{f}\left(\hat{G}^{A} \hat{G}_{1}^{A}-\hat{G}_{1}^{A} \hat{G}^{A}\right) .
\end{aligned}
$$

By making use of the normalization condition $G^{R(A)} G^{R(A)}$ $=1$ and the definition for $\hat{f}$ and $\hat{f}_{1}$ [Eq. (19)], this can be futher rewritten as

$$
\begin{aligned}
\gamma_{B} \xi[ & \left.\frac{d}{d x}\left(f_{L}+\hat{\tau}_{3} f_{T}\right)-\hat{G}^{R} \frac{d}{d x}\left(f_{L}+\hat{\tau}_{3} f_{T}\right) \hat{G}^{A}\right] \\
& +\left(\hat{G}^{R} \hat{G}_{1}^{R}-\hat{G}_{1}^{R} \hat{G}^{R}\right)\left(f_{L}+\hat{\tau}_{3} f_{T}\right) \\
& -\left(f_{L}+\hat{\tau}_{3} f_{T}\right)\left(\hat{G}^{A} \hat{G}_{1}^{A}-\hat{G}_{1}^{A} \hat{G}^{A}\right),
\end{aligned}
$$

which is equal to

$$
\begin{aligned}
\gamma_{B} \xi[ & \left.\left(1-\hat{G}^{R} \hat{G}^{A}\right) \frac{d}{d x} f_{L}+\left(\hat{\tau}_{3}-\hat{G}^{R} \hat{\tau}_{3} \hat{G}^{A}\right) \frac{d}{d x} f_{T}\right] \\
& +\left(\hat{G}^{R} \hat{G}_{1}^{R}-\hat{G}_{1}^{R} \hat{G}^{R}-\hat{G}^{A} \hat{G}_{1}^{A}+\hat{G}_{1}^{A} \hat{G}^{A}\right) f_{L} \\
& +\left[\left(\hat{G}^{R} \hat{G}_{1}^{R}-\hat{G}_{1}^{R} \hat{G}^{R}\right) \hat{\tau}_{3}-\hat{\tau}_{3}\left(\hat{G}^{A} \hat{G}_{1}^{A}-\hat{G}_{1}^{A} \hat{G}^{A}\right)\right] f_{T} .
\end{aligned}
$$

The right-hand side of the last boundary condition in Eq. (A1) can be rewritten with the aid of Eq. (18) into

$$
\begin{aligned}
\hat{G}^{R} \hat{G}_{1}^{R} \hat{f}_{1}-\hat{G}_{1}^{R} \hat{f}_{1} \hat{G}^{A}-\hat{G}^{R} \hat{f}_{1} \hat{G}_{1}^{A}+\hat{f}_{1} \hat{G}_{1}^{A} \hat{G}^{A} \\
\quad-\hat{f} \hat{G}^{A} \hat{G}_{1}^{A}-\hat{G}_{1}^{R} \hat{G}^{R} \hat{f}+\hat{G}^{R} \hat{f} \hat{G}_{1}^{A}+\hat{G}_{1}^{R} \hat{f} \hat{G}^{A} .
\end{aligned}
$$

With Eq. (19) for $\hat{f}$ and $\hat{f}_{1}$ this becomes

$$
\begin{aligned}
& {\left[\hat{G}^{R}\left(\hat{G}_{1}^{R}-\hat{G}_{1}^{A}\right)-\left(\hat{G}_{1}^{R}-\hat{G}_{1}^{A}\right) \hat{G}^{A}\right] f_{L 1}} \\
& \quad+\left[\hat{G}^{R}\left(\hat{G}_{1}^{R} \hat{\tau}_{3}-\hat{\tau}_{3} \hat{G}_{1}^{A}\right)-\left(\hat{G}_{1}^{R} \hat{\tau}_{3}-\hat{\tau}_{3} \hat{G}_{1}^{A}\right) \hat{G}^{A}\right] f_{T 1} \\
& \quad \times\left[\left(\hat{G}^{R}-\hat{G}^{A}\right) \hat{G}_{1}^{A}-\hat{G}_{1}^{R}\left(\hat{G}^{R}-\hat{G}^{A}\right)\right] f_{L} \\
& \quad+\left[\hat{G}^{R} \hat{\tau}_{3} \hat{G}_{1}^{A}-\hat{\tau}_{3} \hat{G}^{A} \hat{G}_{1}^{A}+\hat{G}_{1}^{R} \hat{\tau}_{3} \hat{G}^{A}-\hat{G}_{1}^{R} \hat{G}^{R} \hat{\tau}_{3}\right] f_{T} .
\end{aligned}
$$

Equating left- and right-hand sides of the last boundary condition in Eq. (A1), i.e., Eqs. (A5) and (A7), respectively, finally gives the form of the boundary condition as presented in Eq. (25).

\section{APPENDIX B: TIME CONVOLUTIONS IN ENERGY SPACE}

The expression for a convolution of two functions,

$$
a \circ b\left(t, t^{\prime}\right)=\int_{-\infty}^{\infty} d t_{1} a\left(t, t_{1}\right), b\left(t_{1}, t^{\prime}\right),
$$

can be transformed by changing variables

$$
a \circ b\left(t, t^{\prime}\right)=\int_{-\infty}^{\infty} d t_{1} a\left(t-t_{1}, \frac{t-t_{1}}{2}\right), b\left(t_{1}-t^{\prime}, \frac{t_{1}-t^{\prime}}{2}\right) .
$$

Subsequently, a Fourier transform to energy-frequency space can be made:

$$
\begin{aligned}
a \circ b\left(t, t^{\prime}\right) & =\int_{-\infty}^{\infty} d t_{1} d \omega d E d \omega^{\prime} d E^{\prime} a(E, \omega) e^{i E\left(t-t_{1}\right) / \hbar} e^{i \omega\left(t+t_{1}\right) / 2 \hbar} b\left(E^{\prime}, \omega^{\prime}\right) e^{i E^{\prime}\left(t_{1}-t^{\prime}\right) / \hbar} e^{\left[i \omega^{\prime}\left(t_{1}+t^{\prime}\right)\right] / 2 \hbar} \\
& =\sum_{n^{\prime} n^{\prime}} \int_{-\infty}^{\infty} d t_{1} d E d E^{\prime} a_{n}(E) e^{i E\left(t-t_{1}\right) / \hbar} e^{i n \omega_{0}\left(t+t_{1}\right) / 2 \hbar} b_{n^{\prime}}\left(E^{\prime}\right) e^{i E^{\prime}\left(t_{1}-t^{\prime}\right) / \hbar} e^{i n^{\prime} \omega_{0}\left(t_{1}+t^{\prime}\right) / 2 \hbar} \\
& =\sum_{n^{\prime} n^{\prime}} \int_{-\infty}^{\infty} d E d E^{\prime} a_{n}(E) e^{i E t / \hbar} e^{i n \omega_{0} t / 2 \hbar} b_{n^{\prime}}\left(E^{\prime}\right) e^{-i E^{\prime} t^{\prime} / \hbar} e^{i n^{\prime} \omega_{0} t^{\prime} / 2 \hbar} \delta\left(-E+n \omega_{0} / 2+E^{\prime}+n^{\prime} \omega_{0} / 2\right) \\
& =\sum_{n^{\prime} n^{\prime}} \int_{-\infty}^{\infty} d E^{\prime} a_{n}\left(E^{\prime}+\frac{n+n^{\prime}}{2} \omega_{0}\right) b_{n^{\prime}}\left(E^{\prime}\right) e^{-i E^{\prime}\left(t-t^{\prime}\right) / \hbar} e^{i(n / 2) \omega_{0}\left(t-t^{\prime}\right) / \hbar} e^{i\left[\left(n^{\prime}+n\right) / 2 \hbar\right] \omega_{0}\left(t+t^{\prime}\right)} .
\end{aligned}
$$


With an energy-shift $E=\widetilde{E}+n \omega_{0} / 2$ this becomes

$$
a \circ b\left(t, t^{\prime}\right)=\sum_{n^{\prime} n^{\prime}} \int_{-\infty}^{\infty} d E a_{n}\left(E^{\prime}+\frac{n^{\prime} \omega_{0}}{2}\right) b_{n^{\prime}}\left(E-\frac{n \omega_{0}}{2}\right) \times e^{-i\left[E\left(t-t^{\prime}\right) / \hbar\right]} e^{i\left[\left(n^{\prime}+n\right) / 2 \hbar\right] \omega_{0}\left(t+t^{\prime}\right)} .
$$

The triple products in boundary conditions (3.34) and (3.35) can be worked out in the same manner. The sine and cosine terms cause shifts in the arguments. An example of the result of a triple convolution is given in Eq. (31).

${ }^{1}$ Nonequilibrium Superconductivity, edited by D.N. Langenberg and A.I. Larkin (Elsevier, Amsterdam, 1986).

${ }^{2}$ Nonequilibrium Superconductivity, edited by K.E. Gray (Plenum, New York, 1981).

${ }^{3}$ N.B. Kopnin, Theory of Nonequilibrium Superconductivity (Clarendon, Oxford, 2001).

${ }^{4}$ M. Tinkham and J. Clarke, Phys. Rev. Lett. 28, 1366 (1972).

${ }^{5}$ J. Clarke, in Nonequilibrium Superconductivity (Ref. 1).

${ }^{6}$ G.M. Eliashberg and B.I. Ivlev in Nonequilibrium Superconductivity (Ref. 1).

${ }^{7}$ A.F. Volkov, Phys. Rev. Lett. 74, 4730 (1995).

${ }^{8}$ F.K. Wilhelm, G. Schön, and A.D. Zaikin, Phys. Rev. Lett. 81, 1682 (1998).

${ }^{9}$ S.K. Yip, Phys. Rev. B 58, 5803 (1988).

${ }^{10}$ A.F. Morpurgo, T.M. Klapwijk, and B.J. van Wees, Appl. Phys. Lett. 72, 966 (1998).

${ }^{11}$ J.J.A. Baselmans, A.F. Morpurgo, B.J. van Wees, and T.M. Klapwijk, Nature 397, 43 (1999).

${ }^{12}$ B.J. van Wees, K.-M.H. Lenssen, and C.J.P.M. Harmans, Phys. Rev. B 44, 470 (1991).

${ }^{13}$ P. Samuelsson, V.S. Shumeiko, and G. Wendin, Phys. Rev. B 56, 5763 (1997).

${ }^{14}$ H.T. Ilhan, H.V. Demir and P.F. Bagwell, Phys. Rev. B 58, 15120 (1998).

${ }^{15}$ Th. Schäpers, J. Malindretos, K. Neurohr, S. Lachenmann, A. van der Hart, G. Crecelius, H. Hardtdegen, H. Lüth, and A.A. Golubov, Appl. Phys. Lett. 73, 2348 (1998).

${ }^{16}$ A. Richter, Adv. Solid State Phys. 40, 321 (2000).

${ }^{17}$ P. Samuelsson, J. Lantz, V.S. Shumeiko, and G. Wendin, Phys. Rev. B 62, 1319 (2000).

${ }^{18}$ F. Pierre, A. Anthore, H. Pothier, C. Urbina, and D. Esteve, Phys. Rev. Lett. 86, 1078 (2001).

${ }^{19}$ D. Averin and A. Bardas, Phys. Rev. Lett. 75, 1831 (1995); A. Bardas and D.V. Averin, Phys. Rev. B 56, 8518 (1997).

${ }^{20}$ J.C. Cuevas, A. Martín-Rodero, and A. Levy Yeyati, Phys. Rev. B 54, 7366 (1996).

${ }^{21}$ E.V. Bezuglyi, E.N. Bratus, V.S. Shumeiko, G. Wendin, and H. Takayanagi, Phys. Rev. B 62, 14439 (2000).

${ }^{22}$ A. Brinkman and A.A. Golubov, Phys. Rev. B 61, 11297 (2000).

${ }^{23}$ K.M. Schep and G.E.W. Bauer, Phys. Rev. Lett. 78, 3015 (1997).

${ }^{24}$ Y. Naveh, V. Patel, D.V. Averin, K.K. Likharev, and J.E. Lukens, Phys. Rev. Lett. 85, 5404 (2000).

${ }^{25}$ K.K. Likharev and V.K. Semenov, IEEE Trans. Appl. Supercond. 1, 3 (1991).

${ }^{26}$ M.Yu. Kupriyanov, A. Brinkman, A.A. Golubov, M. Siegel, and H. Rogalla, Physica C 326-327, 16 (1999).

${ }^{27}$ D. Balashov, F.-Im. Buchholz, H. Schulze, M.I. Khabipov, R.
Dolata, M.Yu. Kupriyanov, and J. Niemeyer, Supercond. Sci. Technol. 13, 244 (2000).

${ }^{28}$ A.V. Zaitsev, Pis'ma Zh. Eksp. Teor. Fiz. 51, 35 (1990) [JETP Lett. 51, 41 (1990)]; Pis'ma Zh. Eksp. Teor. Fiz. 55, 66 (1992) [JETP Lett. 55, 67 (1992)].

${ }^{29}$ A.F. Volkov, A.V. Zaitsev, and T.M. Klapwijk, Physica C 210, 21 (1993).

${ }^{30}$ A.V. Zaitsev, A.F. Volkov, S.W.D. Bailey, and C.J. Lambert, Phys. Rev. B 60, 3559 (1999).

${ }^{31}$ S.V. Lempitskii, Zh. Eksp. Teor. Fiz. 85, 1072 (1983) [Sov. Phys. JETP 58, 624 (1983)].

${ }^{32}$ A.M. Kadin, Supercond. Sci. Technol. 14, 276 (2001).

${ }^{33}$ A.F. Volkov and T.M. Klapwijk, Phys. Lett. A 168, 217 (1992).

${ }^{34}$ L.V. Keldysh, Zh. Eksp. Teor. Fiz. 47, 1515 (1964) [Sov. Phys. JETP 20, 1018 (1965)].

${ }^{35}$ J. Rammer and H. Smith, Rev. Mod. Phys. 58, 323 (1986).

${ }^{36}$ A. Schmid in Nonequilibrium Superconductivity (Ref. 2).

${ }^{37}$ G. Schön in Nonequilibrium Superconductivity (Ref. 1).

${ }^{38}$ A.I. Larkin and Yu.N. Ovchinnikov in Nonequilibrium Superconductivity (Ref. 1).

${ }^{39}$ W. Belzig, F.K. Wilhelm, C. Bruder, G. Schön, and A.D. Zaikin, Superlattices Microstruct. 25, 1251 (1999).

${ }^{40}$ A.I. Larkin and Yu.N. Ovchinnikov, Zh. Eksp. Teor. Fiz. 73, 299 (1977) [Sov. Phys. JETP 46, 155 (1977)].

${ }^{41}$ A.V. Zaitsev, Zh. Eksp. Teor. Fiz. 86, 1742 (1984) [Sov. Phys. JETP 59, 1015 (1984)].

${ }^{42}$ M.Yu. Kupriyanov and V.F. Lukichev, Zh. Eksp. Teor. Fiz. 94, 139 (1988) [Sov. Phys. JETP 67, 1163 (1988)].

${ }^{43}$ A.V. Galaktionov and A.D. Zaikin, Phys. Rev. B 65, 184507 (2002).

${ }^{44}$ F. Zhou, P. Charlat, B. Spivak, and B. Pannetier, J. Low Temp. Phys. 110, 841 (1998).

${ }^{45}$ E.V. Bezuglyi, V.S. Shumeiko, and G. Wendin, cond-mat/0303432 (unpublished).

${ }^{46}$ T.T. Heikkilä, J. Särkkä, and F.K. Wilhelm, Phys. Rev. B 66, 184513 (2002).

${ }^{47}$ Th. Schäpers, V.A. Guzenko, R.P. Müller, A.A. Golubov, A. Brinkman, G. Crecelius, A. Kaluza, and H. Lüth, Phys. Rev. B 67, 014522 (2003).

${ }^{48}$ A. Schmid and G. Schön, J. Low Temp. Phys. 20, 207 (1975).

${ }^{49}$ T.T. Heikkilä, T. Vänskä, and F.K. Wilhelm, Phys. Rev. B 67, 100502 (2003).

${ }^{50}$ N. Argaman, Superlattices Microstruct. 25, 861 (1999).

${ }^{51}$ P. Dubos, H. Courtois, O. Buisson, and B. Pannetier, Phys. Rev. Lett. 87, 206801 (2001).

${ }^{52}$ A. Zehnder, Ph. Lerch, S.P. Zhao, Th. Nussbaumer, E.C. Kirk, and H.R. Ott, Phys. Rev. B 59, 8875 (1999). 
${ }^{53}$ S.B. Kaplan, C.C. Chi, D.N. Langenberg, J.J. Chang, S. Jafarey, and D.J. Scalapino, Phys. Rev. B 14, 4854 (1976).

${ }^{54}$ P.C. van Son, J. Romijn, T.M. Klapwijk, and J.E. Mooij, Phys. Rev. B 29, 1503 (1984).

${ }^{55}$ P. Santhanam and D.E. Prober, Phys. Rev. B 29, 3733 (1984).

${ }^{56}$ D.R. Heslinga and T.M. Klapwijk, Phys. Rev. B 47, 5157 (1993).

${ }^{57}$ K.K. Likharev, Dynamics of Josephson Junctions and Circuits (Taylor \& Francis, London, 1992).

${ }^{58}$ E. Bartolomé, A. Brinkman, J. Flokstra, A.A. Golubov, and H. Rogalla, Physica C 340, 93 (2000).
${ }^{59}$ L. Capogna, G. Burnell, and M. Blamire, IEEE Trans. Appl. Supercond. 7, 2415 (1997).

${ }^{60}$ H.H. Zappe, J. Appl. Phys. 44, 1371 (1973).

${ }^{61}$ D. Balashov, F.-Im. Buchholz, H. Schulze, M.I. Khabipov, R. Dolata, M.Yu. Kupriyanov, and J. Niemeyer, Supercond. Sci. Technol. 13, 244 (2000).

${ }^{62}$ H. Sugiyama, A. Yanada, M. Ota, A. Fujimaki, and H. Hayakawa, Jpn. J. Appl. Phys. 36, L1157 (1997).

${ }^{63}$ M. Maezawa and A. Shoji, Appl. Phys. Lett. 70, 3603 (1997). 